\title{
Inferring and Validating Skills and Competencies over Time
}

\author{
Maryam Fazel-Zarandi $\mathrm{a}^{\mathrm{a}}$ and Mark S. Fox ${ }^{\mathrm{b}}$ \\ ${ }^{\text {a }}$ Department of Computer Science, University of Toronto, Toronto, Canada \\ E-mail:mfazel@cs.toronto.edu \\ ${ }^{\mathrm{b}}$ Department of Mechanical and Industrial Engineering, University of Toronto, Toronto, Canada \\ E-mail:msf@eil.utoronto.ca
}

\begin{abstract}
Organizations need to accurately understand the skills and competencies of their human resources to better utilize them and more effectively respond to internal and external demands for expertise. This paper focuses on the problem of inferring and validating skills and competencies over time. In particular, it explores how the quality of often inaccurate, insufficient, and invalid skill and competency information provided by people can be improved. At the core of this work is the development of an ontology for representing and evaluating skills and competencies and capturing information about sources of skill and competency information.
\end{abstract}

Keywords: expert profiling, skills and competency management, human resources management, skills ontology

\section{Introduction}

The ability to accurately locate experts is important to both organizations and knowledge workers. In today's dynamic environment, many companies have transformed their traditionally hierarchical organizations to flatter and flexible structures based around teams (Kodama, 2007) in order to better engage with multiple and changing clients. In such environments, effective resource allocation and team staffing are of utmost importance. Companies need to understand the skills and competencies of their human resources to more effectively respond to internal and external demands for skills and competencies and make more informed hiring decisions. From a knowledge worker's perspective, finding individuals with appropriate skills is important for locating sources of information, accomplishing knowledge intensive tasks, and solving complex problems.

Finding an individual with appropriate knowledge and skills, however, might not be an easy task for many reasons. Expertise is highly dynamic and varies in level (Earl, 2001; Maybury, 2007). When expert involvement in a given activity is required, it is also necessary to know if the expert is actually competent enough to perform the task in addition to being knowledgeable in the field. Furthermore, due to the complexity of some problems, the assistance of multiple experts may be required. Privacy and need-to-know restrictions are other factors that complicate expert finding (D'Amore, 2008). The difficulty of locating experts increases in larger and more geographically distributed organizations and communities. As such, researchers and organizations are increasingly interested in the design and development of automated tools for managing human resource's areas of expertise and performing qualitative and quantitative reasoning about available and required skills and competencies for finding qualified experts.

Effective systems for managing human resources rely significantly on the quality of the data about individuals. To provide the best support, data about human resources must be accurate, up to date, and well defined. To this end, international specifications (e.g., IEEE RCD, 2004; HR-XML, 2006) and automated techniques for construction of expertise profiles of human resources (Stankovic et al., 2010; Yimam-Seid and Kobsa, 2003) have been proposed in recent years. The focus on the syntax of the data exchange and the lack of adequate semantic underpinning (Jarrar et al., 2007), however, have restricted the usefulness of the existing standards. Existing tools are also limited with respect to the sources of information used (D'Amore, 2008) and the ability to represent the changing nature of expertise. Even when starting with 
accurate profiles of individuals, however, they will soon become out-dated. Skills and competencies are dynamic and an individual's knowledge and experience change over time. In addition, individuals often need to acquire new skills due to rapid technological changes in the workplace. As such, it is important to have automated techniques to continually assess, verify, and revise individuals' profiles.

The question we attempt to answer in this paper is starting with less than accurate and incomplete information about an individual, how can we increase the quality of that information and create a model of the individual which can evolve over time. The aspect of information quality relevant to our work is believability which is the extent to which information is regarded as true and credible (Huang, 2008). More specifically, we are interested to know whether a source should be trusted with respect to the information it is providing, whether new information confirms what is already believed about an individual's skills, or if belief should be updated based on the new information. To this end, we use different sources of information in order to reason about individual's skills and competencies in a dynamic environment. To answer the question at hand, we extend the ontology we developed for representing and measuring skills and competencies (Fazel-Zarandi and Fox, 2012) to capture information about sources of skill and competency information. In particular, we focus on how to specify and validate skill statements about an individual over time using different sources of information. In this direction, we specify skills at particular levels of proficiency as what enable the performance of activities, and skill statements as properties that have degrees of belief associated with them and that can change over time. Beliefs in skill statements are then specified and updated based on the credibility of the sources that assert them.

The ontology developed in this paper is used as part of a decision support system for human resources management. The expertise profiles are used to determine whether an individual satisfies a set of requirements for hiring or team staffing, or to conduct gap analysis in order to determine who to train and what training programs to offer. To our knowledge this is a first attempt to model human resources over time by starting with poor information about individuals and transforming it into something good and reliable. One can of course approach the problem of updating beliefs from a Bayesian perspective. We have chosen not to because quantitative information is mostly unavailable. For the little quantitative information that might be available, such as test scores and evaluations, measures coming from different sources would be incomparable. Instead, we take a qualitative approach where skills are defined using a small number of levels with clear and necessary and/or sufficient performance tasks that confirm or refute whether a skill at a specific level has been achieved. As such, this study attempts to understand what types of information are required and how they confirm, refute, or revise what is known about the individual.

The remainder of this paper is organized as follows: Section 2 reviews related work on representing and populating expertise profiles of human resources and highlights the shortcomings of existing approaches. Section 3 presents the methodology used for developing the ontology followed by the ontological framework in Section 4. Sections 5 and 6 describe our approach for creating expertise profiles over time. Section 7 discusses different sources of skill and competency information. Section 8 presents an example and Section 9 discussed the implementation of the ontology. Finally, Section 10 concludes the paper.

\section{Background and Related Work}

The replacement of the traditional system of life-long employment with more flexible work roles (Stevens, 2012), the necessary multi-skilling of workers (Liyanage, 2007), and the increased competition due to globalization have made the management of human assets quite a challenge in recent years. To facilitate this process, competency-based approaches have gained considerable attention. The start of the competency movement within the human resource (HR) literature is credited to an article by McClelland (1973) in which he criticizes the use of intelligence tests in occupational selection and proposes a system of competency-based testing. The advantage of this competency-based approach, as he emphasizes, lies in differentiating good performance from poor performance rather than relying on inferences drawn from certain intelligence factors (Stevens, 2012). Over the years, this approach has gained popularity and today competency management is regarded as an important part of human resources management (HRM) which 
integrates the strategic and operational needs of organizations. Competency management refers to the use of "all instruments and methods used in an organization to systematically assess current and future competencies required for the work to be performed and to assess available competencies of the workforce" (Ley et al., 2007). There are several definitions of competency present in the literature (Draganidis and Mentzas, 2006; De Coi et al., 2007; Stevens, 2012). This term appears to be used at times to refer to actions and their consequences and at others to refer to cognitive skills and personality traits (Stevens, 2012). There have been a number of attempts to bridge the gap between different uses of the term and the majority of recent definitions put emphasis on performance and effectiveness. Campion et al. (2011), for example, define competency as "knowledge, skills, abilities, and other characteristics (KSAOs) that are needed for effective performance in the jobs in question."

The ultimate goal of competency management is to provide the means for ensuring continuous development of organizations' competencies by observing human resources, assessing them according to predefined criteria, and continuously improving them (Urquiza, 2007). As such, it is directly linked to efforts of companies to empower their workforce and leverage internal knowledge in order to increase competitive advantage, innovation, and effectiveness (Draganidis and Mentzas, 2006). More specifically, competency models provide the means for bridging fragmented HR functions such as hiring and promotion decisions, training and development curricula, performance management expectations, employee information management, and organizational change support (Campion et al., 2011; Stevens, 2012). They also improve knowledge management by making explicit what the organization knows and can perform.

In the remainder of this section, we present a review of the research related to modeling and representing skills and competencies, and profiling human resources.

\subsection{Representing Skills and Competencies}

In order to facilitate the interchange of competency descriptions between systems, international specifications such as the Reusable Definition of Competency or Educational Objective (IMS RDCEO, 2002), the IEEE Reusable Competency Definitions (IEEE RCD, 2004), and the HR-XML Competencies (HR-XML, 2006) have been proposed in recent years. These specification provide: (a) identification of the competence, (b) title of the competence, (c) description of the competence, (d) definition of the competence, (e) taxonomy of the competence, (f) personal information (Sampson, 2009). The IMS RDCEO specification aims to enable interoperability among learning systems that deal with competency information and defines competency as "all classes of things that someone, or potentially something, can be competent in." The information stored within most elements of IMS RDCEO are in a string format and as a result they are not directly machine understandable. This limits the potential for systems interoperability. The specification, however, allows the integration of relational information or competence ontologies through embedding additional metadata (Sampson and Fytros, 2008). Another shortcoming of IMS RDCEO is that it does not indicate how competencies are to be assessed, certified, recorded or used. The IEEE RCD specification defines competency as "any aspect of competence, such as knowledge, skill, attitude, ability, or learning objective." This specification describes competencies, as used in a context of learning, education or training, by making direct reference to the IMS RDCEO specification. These definitions are for the purposes of cataloging a competency and not classifying it and can be referenced by external data structures. Similar to IMS RDCEO, IEEE RCD includes information which is for human interpretation.

The above two definitions ignore two important dimensions related to the competency definition, namely, context and proficiency level (De Coi et al., 2007; Prins et al., 2008; Sampson, 2009). The HRXML consortium attempts to extend the previous definition and defines competency as "a specific, identifiable, definable, and measurable knowledge, skill, ability and/or other deployment-related characteristic (e.g. attitude, behavior, physical ability) which a human resource may possess and which is necessary for, or material to, the performance of an activity within a specific business context." Figure 1 illustrates the relevant part of this schema. The HR-XML specification allows the representation of proficiency level information using the CompetencyWeight element. It also makes it possible to record evidence for ranking, comparing, and evaluating the sufficiency or desirability of an individual's competence (HR-XML, 2006). 
However, context is captured only implicitly and is still excluded from the schema (Prins et al., 2008). In addition, although elements for measurable evidence and measurable weights and importance levels are added to the schema, there are no clear semantics of what a value actually means nor are there restrictions on the values of these scales. Furthermore, the connection between competencies and activities are not explicitly represented.

In addition to these three standards, there also exists work on creating thesauri and taxonomies of skills and competencies. The Occupational Information Network ${ }^{1}$ (O*NET-SOC) is one such example that contains occupational definitions but also includes elements of skills and competency measurement. In this effort key features of an occupation are described by a standardized set of variables called "descriptors." The hierarchical model starts with six categories (worker characteristics, worker requirements, experience requirements, occupational requirements, workforce characteristics and occupation-specific information), describing the day-to-day aspects of the job and the qualifications and interests of the typical worker. Another example is DISCO ${ }^{2}$, the European Dictionary of Skills and Competences, a structured, multilingual online thesaurus which is based on existing international standards and classifications and thus represents a terminological basis for the description of skills and competencies, occupations, personal skill profiles and CVs, and job requirements or for describing curricula, courses, or learning outcomes.

The focus on the syntax of the data exchange and the lack of adequate semantic underpinning (Jarrar et al., 2007) have restricted the usefulness of competency standards. More specifically, although these specifications were intended to facilitate the interchange of competency descriptions between systems, they are currently used primarily for human interpretation as they have not focused on semantic interoperability (Sanchez-Alonso and Frosch-Wilke, 2007). As such, in order to enhance the ability of organizations to better utilize their human assets and improve expert finding, it is necessary to extend existing works and address these shortcomings.

Researchers and organizations interested in Expert Finding Systems have also considered different methods of representing skills and expertise of individuals. In this regard both non-ontology-based and ontology-based approaches have been considered. Non-ontology-based approaches use databases as skill repositories in which user profiles are expressed by data structures or weighted vectors of terms (Colucci

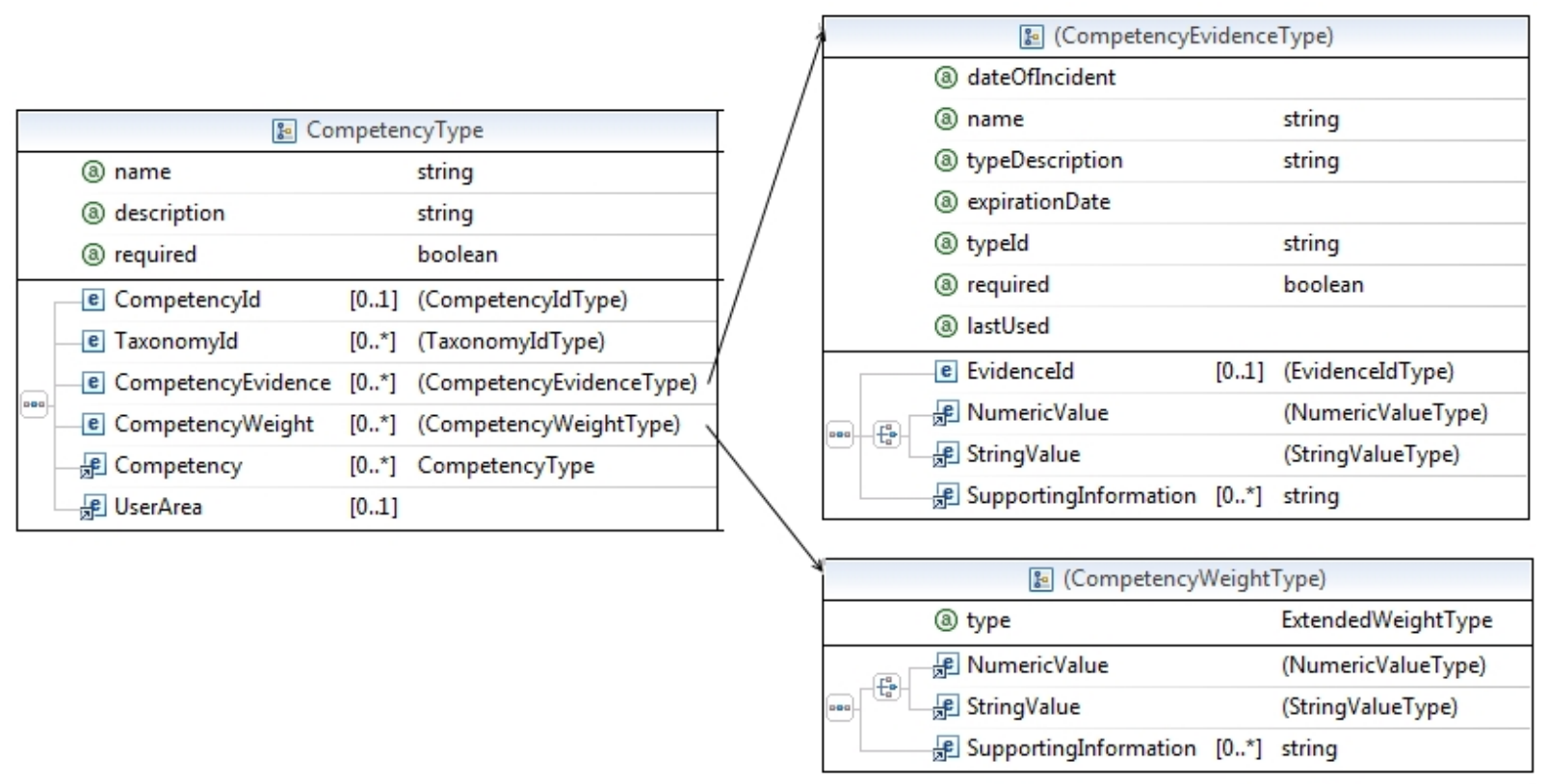

Fig. 1. HR-XML Competencies Schema

\footnotetext{
${ }^{1}$ http://www. onetcenter.org/overview.html

${ }^{2}$ http://disco-tools.eu/disco2_portal/index.php
} 
et al., 2003). The main limitations of these approaches are 1) lack of a common understanding of the terminology used in the organization, and 2) lack of mechanisms for reasoning about individual's skills and competencies.

To address these limitations, the use of formal ontologies for providing a shared common understanding and supporting expertise analysis by reasoning about skills and competencies have been proposed. In the KOWIEN project, research to build up and maintain a detailed ontology-based skill catalog was carried out (Dittmann, 2003). Bizer et al. (2005), Mochol et al. (2007), and Gomez-Perez et al. (2007) develop HR ontologies by integrating existing standards and classifications for supporting the recruitment process by providing an interoperable architecture for e-Employment services. The skills sub-ontology of (Bizer et al., 2005) and (Mochol et al., 2007) is derived from the KOWIEN ontology and provides the means form specifying the competence level of a certain skill. The authors, however, do not discuss the design of the ontology, its concepts and attributes, and the relationship between different sub-ontologies. The HR ontology of (Gomez-Perez et al., 2007) consists of a skill sub-ontology which is based on the European Dynamics Skill classification consisting of 291 skills. This ontology is very simple and only has two concepts of Skill and its subclass ICT Skill and two relations for relating skills to job vacancies and job seekers. Biesalski and Abecker (2006) discuss the integration of HR processes with ontologies in a project at DaimlerChrysler AG. The results are modeled in a competence catalog that represents knowledge over different areas of production, management, and administration. Colucci et al. $(2003 ; 2007)$ use description logic inferences for finding the best individual for a given task based on profiles sharing a common ontology. Although this representation allows the exploitation of an ontological structure for skill management, it does not represent proficiency nor the relation between skills and activities. In other words, it views having a skill as a binary feature and does not specify what it means to have a skill or what can be expected of someone who possess a skill.

The use of ontologies has also been explored in competency-driven e-learning systems and organizational learning. Sicilia (2005), Schmidt and Kunzmann (2006; 2007), Draganidis et al. (2006; 2008), and Dorn et al. (2007) are examples of works that describe ontologies which integrate concepts from competency management and learning objects and resources. The ontology of Dorn et al. (2007) is similar to (Schmidt and Kunzmann, 2006) with the addition of job descriptions composed of required competencies. All of these ontologies support different levels of competencies and the notion of evidences, a concept for showing how a competency was achieved. Schmidt and Kunzmann (2006), however, make a distinction between competencies and competency types, where Competency is an instance of CompetencyType and has a competency level attached to it, whereas a competency types have competency scales. CompetencyType, however, is eliminated from their OWL ontology. A similar distinction is made in (Sicilia, 2005) between the concepts of Competency and CompetencyDefinition, where the former represents "performance capabilities of individuals" and the latter represents "stereotyped descriptions of competencies." This results in a confusion as to what a competency actually is and how it differs from an activity.

An important shortcoming of existing representations is related to competency measurement and evaluation. Rating scales consisting of a numeric scale with a brief description of each number's corresponding meaning are the most widely used for specifying proficiency levels. Their disadvantage, however, lies in their inconsistent interpretations across users of a scale (Moyer, 2001). In addition, it is important to know in each case whether completing a course, for example, is sufficient or actual experience is needed to achieve a certain level of proficiency. Thus, in order to have an objective evaluation mechanism, it is important to associate skills with specific activities and characterize them in terms of measurable and verifiable indicators.

\subsection{Generating Expert Profiles}

There are many different sources of information that can be used for constructing and updating expertise profiles of human resources (Fazel-Zarandi and Yu, 2008; Stankovic et al., 2010). Skill statements can be declared by individuals about themselves or by others, and/or can be derived from 1) activities performed by the individual either online or offline including enrollment in learning activities, experiences related to 
the workplace, generating content both within the organization and on the Web, and question-answering in online forums; 2) recommendations; 3 ) "wisdom of the crowd"; and 4) assessments in the form of various tests, or evaluations in the form of 360 reviews or performance appraisals.

Creating comprehensive profiles of individuals based on their input, however, is a significant challenge. Individuals often lack motivation to add content to their profiles (Hansen et al., 2010) and so over time they become out-dated. Most importantly, self declarations of skills can be incorrect, inaccurate, or insufficient. For example, individuals may exaggerate their competencies for fear of losing their job or they may downplay their proficiency so as not to have more responsibilities (Becerra-Fernandez, 2006). They may also not be aware of the level of their expertise or they may lie on their descriptions of what they contributed or accomplished. As such, manual expert profiling alone is not an effective approach to competency management and expert finding especially in large organizations.

With more activities occurring in the digital environment, implicit and automated expertise extraction from different sources has gained momentum (Stankovic et al., 2010; Yimam-Seid and Kobsa, 2003). Although these systems solve some of the challenges of manually contributing and maintaining expertise profiles, they also introduce new challenges. It is not always easy to relate the author of a particular content with the domains of expertise that it identifies (Stankovic et al., 2010). In addition, some of the individual's expertise may not be represented in their digital trace (Hansen et al., 2010). Furthermore, although generated content may indicate expertise in a field, it is very difficult to determine the level of proficiency of the individual. When considering different sources, it is important to recognize that these sources and the information they provide about skills and competencies vary in degrees of trust, validity, and usefulness. As such, belief in an individual's areas of expertise should be updated as credible new information becomes available. In addition, trust in the sources of information should be revised over time and dishonest behavior in exchanging information about oneself or others should be handled.

In recent years, enterprise social networking and the application of game elements such as point scores, badges, and leader boards to provide motivation for individuals to participate and provide information about the expertise of one another, have also been considered as a different approach to employee profiles (Farrel and Lau, 2006; Bernstein et al., 2009). Some of these platforms, such as LinkedIn ${ }^{3}$, allow individuals to create profiles of themselves and indicate their connections to other users. Others, such as IBM's Fringe Contacts (Farrel and Lau, 2006), allow individuals to describe their colleagues by tagging them with keywords on their expertise and interests, thus, creating a publicly visible tag cloud characterizing the individual (Braun and Schmidt, 2008). The resulting profiles, however, lack commitment by the organization especially with respect to the vocabulary used. Another limitation is the lack of mechanisms for reasoning about individual's skills and competencies and inferring what was not explicitly mentioned.

\subsection{Summary and Discussion}

Proper modeling of skills and competencies provides, among other things, a common language for assessments, a foundation for consistent interviewing, a linkage between performance management and learning, and a means for aligning business strategy and skills for driving organizational performance. As such, it is important to both organizations and knowledge workers. Although over the past decade efforts have gone into the standardization of competency descriptions and improved techniques for human resource evaluation, the process of competency management has been largely human-user oriented. In other words, these specifications are currently used for human interpretation partly due to the fact that they lack adequate semantic underpinning. The use of these specifications in automated expertise profiling is also not clear. Therefore, in order to enhance the ability of organizations to better utilize their human assets and improve expert finding required for accomplishing knowledge intensive tasks, it is necessary to extend existing works and address their shortcomings.

For constructing expertise profiles of human resources, the use of only one source of information is not adequate. When considering different sources, however, it is important to recognize that these sources

\footnotetext{
${ }^{3}$ http://linkedin.com
} 
and the information they provide about skills and competencies vary in degrees of trust, validity, and usefulness. As such, belief in an individual's areas of expertise should be updated as credible new information becomes available. In addition, trust in the sources of information should be revised over time and dishonest behavior in exchanging information about oneself or others should be handled.

A number of formalized representations of skills and competencies have been proposed. These models, however, fall short of addressing some important issues for automated competency management. For example, it is not clear what can be expected of someone who possess a skill, how an individual should be evaluated against a skill, how different sources of information can be used to infer, validate, and update belief in skills and competencies, and how the changing nature of individual's skills should be represented. To answer these questions, further formalization of the semantics related to skill and competency management is necessary. Such formalism will be part of a decision support system for human resources management.

In the remainder of this paper, we present an approach for accurately profiling experts and human resources. In this direction, we first focus on how skills and competencies should be represented and evaluated, and how skill statements about an individual should be modeled. To this end, we develop a formal ontology for representing and reasoning about skills and competencies in a dynamic environment. Furthermore, we identify different sources of skills and competency information and discuss how information from a source can change our belief about the skills of an individual. We extend the ontology with a calculus for updating belief in skill statements based on the credibility of the sources that assert them. In other words, skill evaluations change over time using the axioms as new information becomes available.

\section{Methodology}

For the development of the ontology, we use the ontology design and evaluation methodology of (Gruninger and Fox, 1995). We specify the ontology in four steps: (1) provide a motivating scenario; (2) define informal questions to capture the scenario (i.e. scope); (3) define the terminology (i.e. predicates); and (4) define the axioms (i.e. semantics).

\subsection{Motivating Scenarios}

Organizations need to accurately represent the competency of their human resources in order to better respond to internal and external demands for skills and competencies and make more informed hiring decisions. Wrong decisions in this regard may result in significant loss of value and high turnover of poorly matched human resources (Naveh et al., 2007). This is particularly important for most services organizations, especially those with a medium or large number of employees that provide a variety of products and services to multiple and changing clients. The ability to accurately locate experts in such organizations is also important to knowledge workers. From this perspective, finding individuals with appropriate skills is important for accomplishing knowledge intensive tasks and solving complex problems.

As discussed in Section 2, proper modeling of skills and competencies and accurately profiling human resources provides the means for bridging fragmented HR functions such as hiring and promotion decisions, training and development curricula, performance management expectations, employee information management, and organizational change support (Campion et al., 2011; Stevens, 2012). More specifically, related use cases range from locating and matching individuals and requirements, to composing teams, conducting gap analysis, and human resource development, and include activities such as queries about individual human resources and teams of experts, development history, and believability of a certain piece of information. In the following we consider two use case scenarios. These use-cases are derived from the relevant literature and structured interviews of two HR professionals.

Activity Assignment: Suppose that Mary is a project manager working at an IT consulting company, responsible for selecting individuals to work on specific projects for industry clients. This is an important responsibility with severe consequences if poor assignments are selected: it may result in project failure 
due to time, cost, or quality, loss of customers, loss of referrals from a customer, employee dissatisfaction and attrition, and loss of revenue (Naveh et al., 2007). To do her job well, Mary needs to know the competencies of available human resources to match them with the existing requirements, and determine if the company needs to hire a new employee or outsource the project. To this end, she needs to gather skills and competency related information about individuals and judge whether they are relevant to the requirements. In order to identify the best person for the job, Mary considers different sources of information. It is often the case, however, that the required information is either not explicitly available, is unreliable, or out-of-date (Becerra-Fernandez, 2006; Naveh et al., 2007; Hansen et al., 2010; Hensel et al., 2010). The same process also applies, more or less, to the problem of outsourcing work to other companies or a crowd where a set of individuals perform assigned activities on demand.

Human Resource Recruitment: Assume that for one of the projects, Mary needs to hire a new employee. A usual recruitment process includes four phases (Bizer et al., 2005): 1) requirements analysis, 2) publishing the job posting, 3) receiving and preselecting applications, and 4) final recruitment decision. As such, Mary posts a job advertisement on the company website and asks all potential candidates to submit their resume and provide references. Once an applicant pool has been formed, Mary preselects a group of candidates by grading them according to the degree of match to the skill and competency requirements of the job posting and their references. The difficulty in this stage is in matching and relating individual's declared skills and competencies, acquired and assessed skills and competencies as suggested by their credentials and certificates, and applied skills as suggested by their previous work experience to job requirements (Bizer et al., 2005; Mochol et al., 2007; Fazel-Zarandi and Fox, 2009). For example, the same position title in two different companies may refer to different responsibilities and set of skills. In verifying individual's competencies, references may also be dishonest in exchanging information or not sufficiently competent or consistent in providing useful and accurate evaluations (Tommasi et al., 1998; Nicklin and Roch, 2009). Once Mary selects the viable candidates, she asks them to attend an interview. The interview is composed of two parts: a discussion phase where one or more employees have the opportunity to ascertain the interviewee's skills and competencies through direct question and answering, and a testing phase where the interviewee is asked to perform one or more tasks that demonstrate the level of skills they possess.

These use cases may seem very different from the point of view of human resources management. However, central to both scenarios is the importance of gathering, representing, and validating useful skill and competency related information from different sources in order to match them to the requirements.

\subsection{Informal Competency Questions}

Competency questions specify the requirements of the ontology (Gruninger and Fox, 1995) and the types of queries that are required for supporting HR decision making. These are questions that impose demands on the expressiveness of the ontology (Katsumi and Gruninger, 2010) and the ontology should be able to represent and answer these questions using a necessary and sufficient set of axioms (Gruninger and Fox, 1995; Katsumi and Gruninger, 2010).

The key concepts abstracted from the scenarios described in the previous section are the following:

C-1 There must be a systematic way of identifying skill requirements for successful performance of the required activities and determining whether an individual possesses a particular skill.

C-2 The ability of individuals to perform the activities enabled by a particular skill may vary. There must be a systematic way of assessing activity performance and evaluating the quality of outcomes produced.

C-3 Different sources of information about individual's skills and competencies vary in degrees of trust and validity. There must be a systematic way of identifying credible sources of information.

C-4 The degree to which a statement about an individual's skill can be believed depends on the evidence that supports it. There must be a systematic way of changing belief in skill statements over time as new information becomes available. 
C-5 Individuals are not always honest in exchanging information about themselves or others, and may engage in collusive behaviors with others.

In this paper, we focus on $\mathrm{C}-1$ to $\mathrm{C}-4$. To elaborate $\mathrm{C}-1$ and $\mathrm{C}-2$, we need to focus on the connection between skills and activity performance and the relation between skills. A skill suggests the possibility of performing an activity. The following are informal competency questions about skills:

Q-1 What skills are needed to perform the required activities?

Q-2 Are two skills related? Does having one imply having the other?

Not everyone possessing a skill, however, can perform all the activities it enables, thus, it is also necessary to specify the level of proficiency that is required. Proficiency in a skill may depend on different factors such as familiarity with the subject, the span of the activities that one has performed, how much experience one has in performing the activities, etc. Different measurement scales exist for evaluating an individual against a skill. Rating scales, consisting of a numeric scale with a brief description of each number's corresponding meaning, are the most widely used. Their disadvantage, however, lies in their inconsistent interpretations across users of a scale (Moyer, 2001). Domain-specific metrics, such as number of errors found in a code for a programmer or number of failed projects for a contractor, are other metrics that can be used. The following are some questions for representing and measuring proficiency:

Q-3 What are the proficiency reference levels for evaluation against a skill?

Q-4 What are the criteria for determining whether an individual possesses a skill at a level of proficiency?

Q-4.1 What are the activities that the individual should be able to perform?

Q-4.2 What are the attributes related to that skill that can be measured?

Q-4.3 What is the unit of measurement for an attribute related to a skill?

Q-4.4 What ought to be the measured value to be ranked at a level of proficiency?

In order to elaborate $\mathrm{C}-3$, we need to take into account the different sources of information that can be used to infer and validate individual's skills and competencies. The following are some questions about skill statements about individuals:

Q-5 What evidence suggests that an individual has a skill at a level of proficiency?

Q-6 Which source is providing this evidence? Should it be considered as a credible source of information?

To elaborate C-4, we need to identify the skill statements of an individual at a given point in time and indicate how degree of belief in the skill statement changes over time:

Q-7 What are the skill statements about an individual at a given point in time?

Q-7.1 What are the demonstrated skills of an individual at a given time point?

Q-7.2 What are the suggested skills of an individual at a given time point, i.e. those skills that have not been observed but the individual may possess?

Q-7.3 What are the refuted skills of an individual at a given time point, i.e. those skills that the individual does not have?

Q-8 How did belief in a skill statement change over time?

\section{Ontological Framework}

We use first-order logic (FOL) as the basis of our representation because of its expressive and declarative capability. The use of FOL instead of more limited representations (e.g., OWL-DL, Horn clauses) makes it possible to compactly represent and reason about skills and competencies over time. The use of OWL-DL, for example, would make it hard to encode and reason about processes and events in addition to limiting us to binary relations (Kuhn, 2010). In fact, description logics have been mostly used to describe 


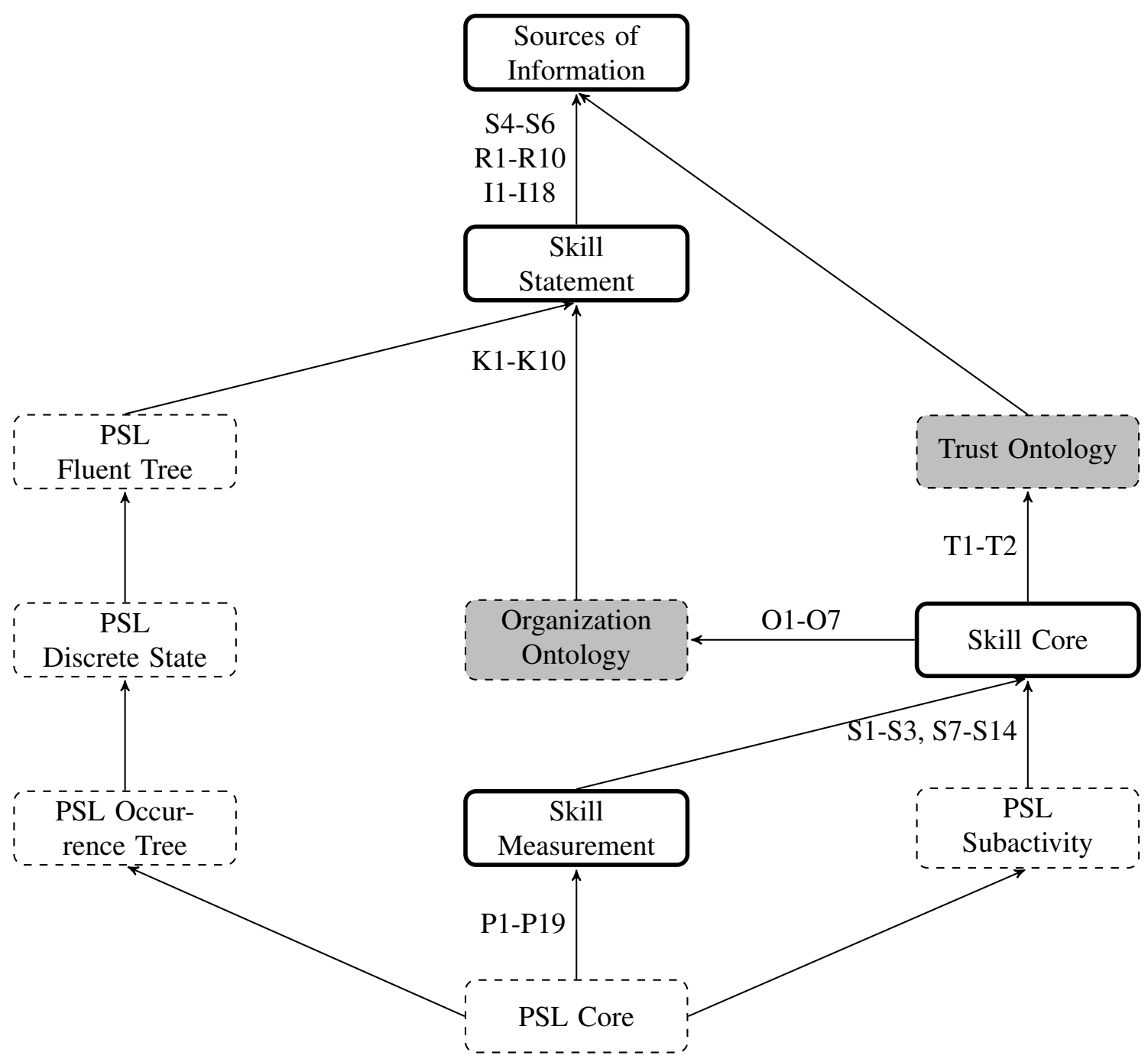

Fig. 2. Modules of the Ontology for Skill and Competency Management. Shaded modules indicate modified ontologies.

static knowledge-base systems (Gu and Soutchanski, 2010). However, in order to infer and validate skills and competencies and change belief in the skills and competencies of individuals dynamically as new information is gathered, we need to encode and reason about processes and activities. To specify the preconditions and results of processes, however, we need expressiveness comparable to that of FOL (Martin et al., 2007).

Our Ontology of Skill and Competency Management is organized into sub-ontologies or modules (Figure 2). In particular, our ontology is an extension of the Process Specification Language (PSL) (Gruninger and Menzel, 2003) which provides predicates and axioms that enable representation of and reasoning about fluents ${ }^{4}$, activities, activity-occurrences, and values of fluents before and after activity-occurrences. For example, the Activity-Occurrence Extension of PSL defines relations that allow the description of how activity-occurrences relate to one another with respect to the time at which they start and end; and the State Extension introduces the concept of state (before an activity-occurrence) and post-state (after an activity-occurrence). Table 1 presents the PSL predicates used in this paper. In the formal PSL ontology, the notion of activity is a basic construct, which corresponds intuitively to a kind of activity. An activity

${ }^{4}$ Fluents are properties of the real world that can change over time. There are two types of fluents: relational fluents refer to relations that have true values of "true" or "false"; and functional fluents refer to the functions as defined in mathematics (represented as terms) (Huang, 2008). 
Table 1

PSL predicates used in this paper (Gruninger and Menzel, 2003)

\begin{tabular}{|l|l|}
\hline \multicolumn{1}{|c|}{ Predicate } & \multicolumn{1}{c|}{ Informal Definition } \\
\hline activity $(A)$ & $\begin{array}{l}\text { A class or type of action. Intuitively, activities can be considered to be } \\
\text { reusable behaviors within the domain. }\end{array}$ \\
\hline activity-occurrence $(O)$ & $\begin{array}{l}\text { An event or action that takes place at a specific place and time. An in- } \\
\text { stance or occurrence of an activity. }\end{array}$ \\
\hline object $(X)$ & $\begin{array}{l}\text { Anything that is not a timepoint, nor an activity, nor an activity- } \\
\text { occurrence. }\end{array}$ \\
\hline state $(F)$ & $\begin{array}{l}\text { Intuitively, states represent properties and relationships in the domain } \\
\text { that can change as the result of the occurrence of activities. }\end{array}$ \\
\hline timepoint $(T)$ & A point in time. \\
\hline occurrence-of $(O, A)$ & The activity-occurrence is a particular occurrence of the given activity. \\
\hline subactivity $\left(A_{1}, A_{2}\right)$ & $\begin{array}{l}\text { This relation defines a partial ordering over the set of activities with } \\
\text { respect to aggregation and decomposition. }\end{array}$ \\
\hline before $\left(T_{1}, T_{2}\right)$ & This relation is used to impose a total ordering on timepoints. \\
\hline endof $(O)$ & The timepoint at which the occurrence ends. \\
\hline holds $(F, O)$ & The state is true after the activity-occurrence. \\
\hline prior $(F, O)$ & The state is true before the activity-occurrence. \\
\hline achieved $(F, O)$ & $\begin{array}{l}\text { A state is achieved by the activity-occurrence if and only if it does not } \\
\text { hold before the occurrence, but it does hold after the occurrence. }\end{array}$ \\
\hline falsified $(F, O)$ & $\begin{array}{l}\text { A state is falsified by the activity-occurrence if and only if it holds before } \\
\text { the occurrence, but does not hold after the occurrence. }\end{array}$ \\
\hline
\end{tabular}

may have associated occurrences, which correspond to individual instances or executions (from start to finish) of the activity.

Additionally, we reuse parts of the Organization Ontology (Fox et al., 1996) and the Measurement Ontology (Kim et al., 1999). The Organization Ontology was developed as part of the Toronto Virtual Enterprise (TOVE) project (Fox and Gruninger, 1998) in cooperation with several companies with the goal of providing a basis for enterprise modeling. An organization, as viewed in TOVE, is a set of constraints on the activities performed by agents (Fox et al., 1996). An Organization consists of a set of divisions and subdivisions, a set of agents, a set of roles that the members play in the organization, and a set of goals that the members try to achieve. We also use the Trust Ontology presented in (Huang, 2008; Huang and Fox, 2006) which formally defines the semantics of information sources, information dependencies, relationships between information sources and experts, and trust relationships. The latter two ontologies are particularly useful for inferring skills and competencies and will be discussed in more detail in the following sections.

In the remainder of this section, we briefly present the basic elements of the ontology required for inferring, assessing, and validating skills and competencies over time by considering different sources of information. These elements are used to state what a skill is, what can be expected of someone who possesses a skill, and how an individual should be evaluated against a skill. For brevity, not all axioms are presented here. The interested reader is referred to (Fazel-Zarandi and Fox, 2012).

\subsection{Skills and Proficiency Levels}

For describing human resources, we consider skill as the core element that is necessary for performing certain activities in the workplace. Furthermore, we consider expertise and competency as indicating the level of performance of the related activities (e.g., competency indicates sufficiency of skills or knowledge, 
Table 2

Predicates used for representing and measuring skills and competencies

\begin{tabular}{|l|l|}
\hline \multicolumn{1}{|c|}{ Predicate } & \multicolumn{1}{c|}{ Informal Definition } \\
\hline skill $(S)$ & $\begin{array}{l}\text { A class or type of skill. A skill suggests the possibility of per- } \\
\text { forming an activity. }\end{array}$ \\
\hline proficiency-level $(L)$ & $\begin{array}{l}\text { Refers to the ranking of the ability of an individual to perform } \\
\text { the activities enabled by a particular skill. }\end{array}$ \\
\hline measured-attribute $(M)$ & A measurable attribute related to a skill. \\
\hline specification-set $(S p)$ & $\begin{array}{l}\text { A set of values which denotes possible values for a measured- } \\
\text { attribute. }\end{array}$ \\
\hline measurement-unit $(U)$ & Unit of measurement for a measured-attribute. \\
\hline enables $(S, A, L)$ & Skill $S$ enables activity $A$ at level of proficiency $L$. \\
\hline enabling-suite $(S, A, L)$ & $\begin{array}{l}\text { A complex activity that includes all the activities enabled by a } \\
\text { skill at a particular level of proficiency. }\end{array}$ \\
\hline in-field $(S, F)$ & Skill $S$ is in knowledge-field $F$. \\
\hline dominates $\left(L_{1}, L_{2}\right)$ & Proficiency level $L_{1}$ dominates level $L_{2}$. \\
\hline requires-value $(S, L, M, X)$ & $\begin{array}{l}\text { Skill } S \text { at level of proficiency } L \text { for measured attribute } M \text { re- } \\
\text { quires value } X \text {. }\end{array}$ \\
\hline performs $(P, O)$ & $P$ performs the activity-occurrence. \\
\hline
\end{tabular}

i.e., the minimum level of acceptable performance (Herling, 2000), whereas expertise indicates superior skills or knowledge in a particular area, i.e., the optimal level of performance.). More specifically, we specify skills as what enable the performance of activities, and relate proficiency in a skill to the span of activities that one can perform in addition to measurable attributes related to that skill. Table 2 presents the required predicates for representing and measuring skills and competencies. The overall relations between skills, proficiency levels, and activities are illustrated in Figure 3. The notion of activity in PSL is a basic construct, which corresponds intuitively to a kind of activity. An activity may have associated occurrences, which correspond to individual instances or executions (from start to finish) of the activity. Occurrences are related to an activity by the binary predicate occurrence-of. In the following, we define these concepts and the relations between them for representing and measuring skills and competencies.

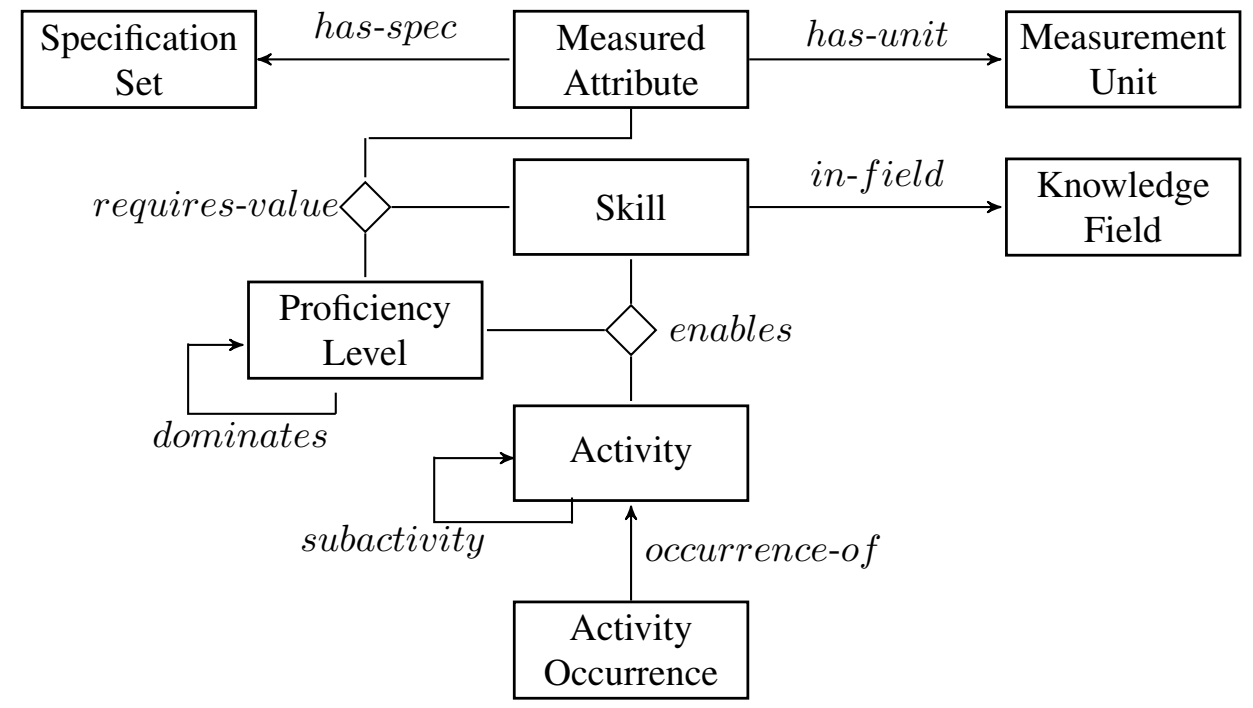

Fig. 3. Concepts and their relations in the static representation 
A skill at a particular level of proficiency enables a set of activities:

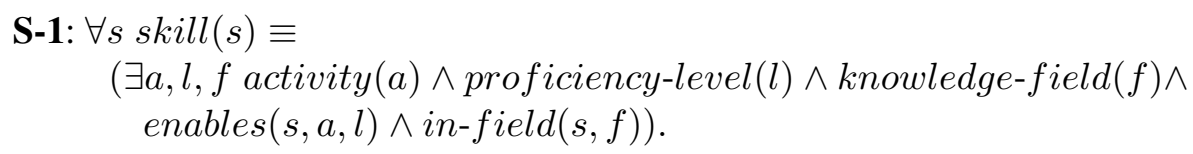

The in-field predicate relates a particular skill to the knowledge-field (context of the skill) that it belongs to. Level of proficiency refers to the ranking of the ability of an individual to perform the activities enabled by a particular skill. For example consider the software development skill. Some of the high level core activities enabled by this skill are read code, write code, design software, use libraries and frameworks, debug code, use version control, perform unit testing, revise existing code, use build automation, etc. Not everybody having this skill can perform all of these activities sufficiently well, therefore, it is important to specify the level of proficiency. We consider proficiency as determined by the successful performance of activities the skill enables. We define the ordering between proficiency-levels by the dominates relation which is transitive and asymmetric. If an individual has the skill at a level of proficiency, then she can perform all the activities associated with the skill at lower levels. The minimum level of proficiency for a skill $s$ (min-level $(s, l)$ ) is the level that is dominated by all other proficiency levels for this skill but does not dominate any level itself.

As mentioned earlier, in addition to the span of activities that one can perform, we also consider attributes related to a skill that can be measured (measured-attribute). For example, recency, years of experience, the time it takes to complete activities, and average number of errors found in a source code are some of the attributes that can be used to measure proficiency in software development. This provides the means for reducing fluctuations in competency measurement. To this end, we use ideas from the Measurement Ontology (Kim et al., 1999). Measured attributes are input to the model (primitive types), and can be categorical or numeric. Each measured-attribute takes up values from a specification set (has-spec) which is either defined by its elements and an ordering between the elements, or over an interval. All measured attributes must have a unique specification set and a unit of measurement. Each measured-attribute is measured through a series of activities that perform measurement and the result is represented as a measurement point (measurement-pt). The required value of a particular measured-attribute $m$ related to a skill $s$ is identified for different levels of proficiency $l$ using requires-value(s, $l, m, x)$, where $x$ is the required value. For example, considering recency as an attribute for measuring proficiency in software development, to be ranked as having the skill at level competent, the value of less than two years is required. The required value for an attribute at a level of proficiency is unique and must be an element of its specification set. If a value is required for a measured attribute at a level of proficiency, the same or a higher value is required for higher levels.

In many domains of interest, it is also possible to assume a taxonomy of skills with specialization of skills defined using the subskill-of relation. This relation is reflexive, transitive, and asymmetric. A skill enables all the activities its super skill enables at the same or higher level of proficiency, and inherits the required values of its parent for a measured attribute, if it does not explicitly have a required value assigned. We also define the related-to relation between two skills indicating that they are highly related to each other in the domain of interest. For example, database development skill is related-to programming skills since they come together in software design and development. More specifically, the database development skill enables data modeling required for designing software, and programming skills enable reading, writing, and debugging code. Put formally, two skills are related if they enable the same activity, or if they enable different subactivities of the same activity.

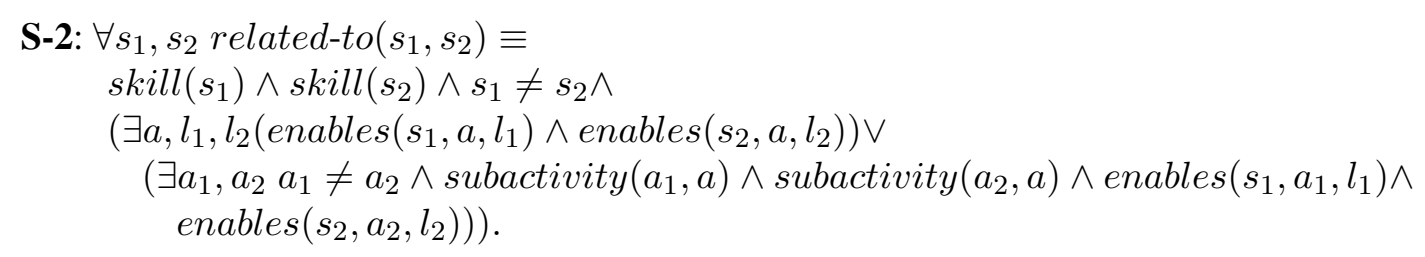


The related-to relation is symmetric. Furthermore, a skill is related to the skills that its super skill is related to. These relations can be used to infer further knowledge about individuals' skills and competencies in the absence of explicit data.

\subsection{Skill Statements}

The unit of skills information about the individual that we consider is a skill statement which states that "individual $p$ has skill $s$ at level of proficiency at least $l$." Each human resource will have a profile composed of a set of such skill statements. Existing standards and representations view having a skill as a binary feature, i.e., an individual either has or does not have a particular skill (see Section 2). However, for representing the skills and competencies of human resources this is not adequate since different sources are used to construct expertise profiles. More specifically, the degree to which we can believe a skill statement depends on the source from which it was derived and the evidence that supports it. For example, in many organizations workflow management systems are used to track employees' daily activities. If it is recorded that an individual has performed all the activities which a skill enables at a level of proficiency, then we can be sure that the individual has the skill. On the other hand, skill statements asserted by individuals about themselves may not be as accurate and reliable. As such, when constructing expertise profiles, we need to take into account the extent to which information about the individual can be regarded as true and credible. In other words, we need to differentiate between different kinds of evidence in support of a skill statement based on the credibility of the source that is asserting it in order to discount uncertainty.

To represent the believed truth value of a skill statement, we chose not to use a probabilistic model as the available information was not reducible to probabilities; references, test results and other sources of support could not be measured on a rational scale due to variations in their source (i.e., who wrote it, who tested it). Hence we had to consider an ordinal scale. It was clear that the information could be categorized into that which demonstrably confirmed (i.e., via tests or activities at the hiring organization) or refuted the possession of a skill, or that which was purely suggestive. A skill statement is True if the person has demonstrated the skill it refers to and is False if the individual has failed an activity or a measured attribute related to that skill. When a skill statement is demonstrated to be True, we refer to it as a demonstrated skill statement, and when it is demonstrated to be False, we refer to it as a refuted skill statement. To handle skill statements that have not been demonstrated to be True or False, we rely upon evidence based on the credibility of sources that assert them and define two values of probable and possible. The value of possible refers to a situation where the skill has not been demonstrated False, nor is there any other evidence from a credible source to support it. The value of probable indicates that credible sources are suggesting a skill statement. To represent the believed truth value of a skill statement, we use the PSL discrete state extension. At any point in time a skill statement can be in one and only one of four states: demonstrated, probable, possible, or refuted (Table 3). These states are core to how the understanding of skills evolves. Starting with less than accurate information about the individual, skill statements can be suggested or refuted as information becomes available until they are demonstrated. We will discuss credibility of sources and the information they provide in more detail in the next section.

To see how skill evaluation changes over time, consider the simple example of Mary declaring that she has the object-oriented programming skill at level of proficiency $l$. Since this is a self-declaration, we can only acknowledge that she possibly has the skill. However, if we know that she has previous experience as Java Developer which requires object-oriented programming at least at level $l$ and her former manager recommends her, we can infer that most likely she has the skill. As more information becomes available on activities performed by Mary, proficiency is measured and it is noticed that she does not meet one of the required criteria for having the skill at level $l$, thus, the skill statement is refuted. Nevertheless, Mary may have the skill at a lower level of proficiency. Thus, a statement referring to this skill at a lower level is added to the knowledgebase. Since she has performed all the activities required for this level, we can be sure that Mary in fact has the skill at the specified level.

To this end, the states extension of PSL provides the concepts for relating states of the world to activity occurrences. If a skill statement at a particular level of proficiency is in any of the states except for refuted, 
Table 3

States of skill statements

\begin{tabular}{|l|l|}
\hline \multicolumn{1}{|c|}{ Fluent } & \multicolumn{1}{c|}{ Definition } \\
\hline demonstrated $($ skill-statement $(p, s, l))$ & $\begin{array}{l}\text { Relational fluent. } p \text { has demonstrated skill } s \text { at level of pro- } \\
\text { ficiency at least } l \text {. }\end{array}$ \\
\hline probable(skill-statement $(p, s, l))$ & $\begin{array}{l}\text { Relational fluent. It is highly probable that } p \text { has skill } s \text { at } \\
\text { level of proficiency at least } l \text {. }\end{array}$ \\
\hline possible $($ skill-statement $(p, s, l))$ & $\begin{array}{l}\text { Relational fluent. It is possible that } p \text { has skill } s \text { at level of } \\
\text { proficiency at least } l \text {. }\end{array}$ \\
\hline refuted $(\operatorname{skill-statement}(p, s, l))$ & Relational fluent. $\operatorname{skill-statement}(p, s, l)$ has been refuted. \\
\hline asserted $(\operatorname{skill-statement}(p, s, l))$ & $\begin{array}{l}\text { Relational fluent. } \text { skill-statement }(p, s, l) \text { is demonstrated, } \\
\text { probable, or possible. }\end{array}$ \\
\hline
\end{tabular}

then it is also in that state at lower levels if no information about lower level is available. On the other hand, if a skill statement is refuted at a level of proficiency, it is also refuted at higher levels. If a skill statement is in any of the states except for refuted then it is asserted. Furthermore, if the state of a skill statement changes, its previous state does not hold any longer.

\section{Demonstration and Refutation of Skills}

The best way to verify whether individuals actually possess a skill is to observe how they perform on the job. Even prior to hiring, job simulations can be performed in which applicants are given a realistic job related situation and evaluated on their performance. If an individual is observed to perform all the activities which a skill enables at a level of proficiency and satisfies all the measured attributes, then it is the case that he or she has demonstrated that skill. On the other hand, if the individual does not satisfy at least one measured attribute, then the skill is refuted at the corresponding level.

Here, the order in which the activities have been performed is not important, and once all the enabled activities have been performed, the conclusion can be made. As such, we define the enabling-suite of a skill at a level of proficiency (i.e., the complex activity including all the activities enabled by that skill at this particular level) as:

S-3: $\forall s, l, a$ enabling-suite $(s, a, l) \equiv\left(\forall a_{1}\right.$ enables $\left.\left(s, a_{1}, l\right) \supset \operatorname{subactivity~}\left(a_{1}, a\right)\right)$.

Note that in PSL, each complex activity occurrence has a unique atomic root occurrence and each finite complex activity occurrence has a unique atomic leaf occurrence. An occurrence is the leaf of an activity tree if and only if there exists an earlier atomic subactivity occurrence but there does not exist a later atomic subactivity occurrence.

Using the definition for enabling-suite, and considering the attributes that are measured and the actual measured values (i.e., measurement point denoted by measurement-pt) for an agent, we can write the above intuition. If an individual is observed to perform all the activities which a skill enables at a level of proficiency and satisfies all the measured attributes, then it is the case that he or she has demonstrated that skill:

S-4: $\forall o, p, s, l, a$

$$
\begin{aligned}
& (\text { enabling-suite }(s, a, l) \wedge \text { leaf }(o, a) \wedge \\
& \left.\quad\left(\forall a_{1} \text { subactivity }\left(a_{1}, a\right) \supset\left(\exists o_{1} \text { occurrence-of }\left(o_{1}, a_{1}\right) \wedge \operatorname{performs}\left(p, o_{1}\right)\right)\right)\right) \wedge \\
& \left(\forall m, x \text { requires-value }(s, l, m, x) \supset\left(\exists x_{1} \text { measurement-pt }\left(p, m, x_{1}\right) \wedge \operatorname{lesser} E q\left(x, x_{1}\right)\right)\right) \\
& \supset \text { achieved }(\text { demonstrated }(\operatorname{skill}-\text { statement }(p, s, l)), o) .
\end{aligned}
$$

where, leaf $(o, a)$ is true when $o$ is a leaf of the activity tree for $a$ as defined in the formal PSL ontology. An activity tree consists of all possible sequences of atomic subactivity occurrences beginning from a root subactivity occurrence. A fluent is achieved by an activity occurrence if and only if it does not hold before 
the occurrence, but it does hold after the occurrence. If the fluent already holds, i.e., the skill statement is in state demonstrated, then nothing changes. If, however, this is not the case, i.e., the skill statement is in one of the other states or no information is available about the skill, then the state is changed to demonstrated once all the activities enabled by a skill are performed by the individual and he or she satisfied all the measured attributes. Here fluents are represented in the reified form and as such may have other fluents as parameters.

If the individual has performed occurrences of all activities enabled by a skill, but the related attributes have not yet been measured, the state of the skill statement is set to probable:

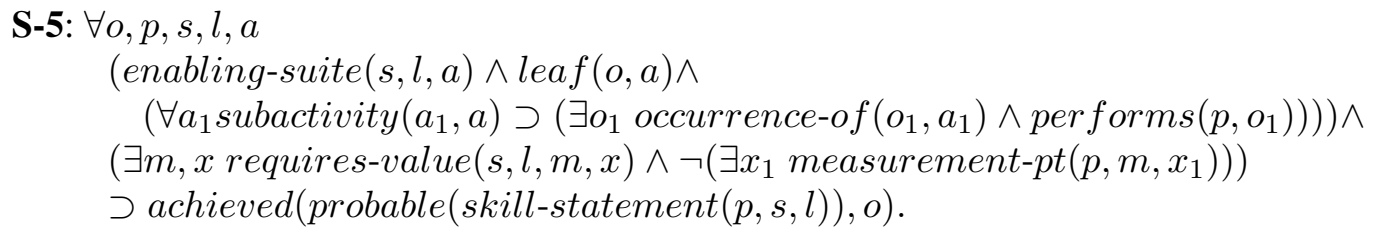

On the other hand, if the individual does not satisfy at least one measured attribute related to a skill, then the skill is refuted at the corresponding level. This state is not reversible by information provided by other sources of skill and competency information (discussed in more detail in the next section). However, if at a later point in time the individual is measured again and satisfies the required value, then depending on other related attributes and activities, the state can be changed to demonstrated using S-4.

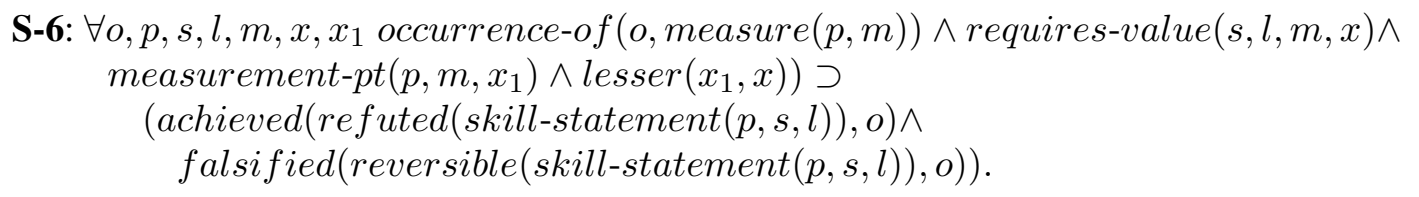

\section{Modifying Skill Evaluations Over Time}

Observing individuals to determine their skills and competencies is costly and can be time consuming. More specifically, prior to hiring, job simulations are not suited to all jobs and are limited in scope in that they can only focus on a subset of activities and duties (McPhie, 2010). In addition, depending on the job type, work simulations may require time and expertise to develop and update suitable exercises and to administer and assess applicants, and/or may consume job-related resources and equipments (McPhie, 2010). There is also a high cost to hiring and then firing an unqualified individual for the job. As such, it is important to use other sources of skill and competency information to construct expert profiles and to better understand and validate skills and competencies of individuals over time. In doing so, however, it is important to recognize that these sources and the information they provide vary in degrees of trust and validity, respectively. For example, self declarations of skills can be incorrect, inaccurate, or insufficient. In verifying individual's skills and competencies, references may also be dishonest in exchanging information or not sufficiently competent to provide accurate evaluations. Assessment results may also lack validity and reliability due to cognitive biases (Seta et al., 2005). Furthermore, studies have shown that it is often the case that the assessment process is unstructured, inconsistent, and lacks specified criteria (Fernandez-Araoz et al., 2009). Information provided by different sources may also be from different views and therefore conflicting. As such, when considering other sources, a challenge is to judge which information is more credible.

The concept of credibility has been studied by researchers in diverse fields for many years. Despite this fact, a clear, agreed upon definition of credibility is lacking (Hilligoss and Rieh, 2008). Credibility has been defined as believability, trust, perceived reliability, accuracy, objectivity, and many of other concepts and combinations of them (Self, 1996; Hilligoss and Rieh, 2008). It has also been defined in terms of the characteristics of the person, organization or medium, and the information and message offered (Self, 1996). Researchers, however, generally agree that credibility assessment is done through evaluating multiple dimensions, and the vast majority of scholars identify trustworthiness and expertise as the 
two key components of credibility (Tseng and Fogg, 1999). These two dimensions can be connected by considering trust in a particular field of knowledge.

In order to represent the credibility of a source, we use formal semantics of trust defined in (Huang, 2008; Huang and Nicol, 2009). In particular, the trusted-in predicate is used which denotes that a trustor (the organization in our case) trusts an information creator on producing information in a particular knowledge field at particular point in time. This predicate corresponds to an external process to make trust judgments. If the trust relationship holds, then process returns true, otherwise returns false. Additionally, distrusted-in means the trustor believes that the information provided by the individual is false. The reason for introducing this predicate is that distrust is a negative form of trust and not the negation of trust (Marsh and Debben, 2005) $)^{5}$. If a source is trusted in the related field at a particular point in time, then it is considered as a credible source for that skill (T-1). If, on the other hand, the source is distrusted, then the source is not credible (T-2).

T-1: $\forall x, f, t, s$ trusted-in $(x, f, t) \wedge$ in-field $(s, f) \supset$ credible-source-for $(x, s, t)$.

T-2: $\forall x, f, t, s$ distrusted-in $(x, f, t) \wedge$ in-field $(s, f) \supset \neg$ credible-source-for $(x, s, t)$.

It is important to note that credibility assessment is an iterative process and the belief in the credibility of a source may change over time. For example, we may start with a set of trust statements about individuals and institutions, or the belief that a test reliably measures a skill. Over time, however, we may discover that these assumptions were not accurate to begin with or are no longer valid. In this case, it is necessary to modify trust in such a source. We do not address this issue in this paper ${ }^{6}$. Here, we only note that if a source is trusted at time $t_{1}$ and distrusted at time $t_{2}, t_{1}<t_{2}$, then at some point in time between $t_{1}$ and $t_{2}$ trust was revised in this source. This is required for revising belief in skill statements asserted by a distrusted source, discussed later in this section.

T-3: $\forall x, f, t_{1}, t_{2}$ trusted-in $\left(x, f, t_{1}\right) \wedge$ distrusted-in $\left(x, f, t_{2}\right) \wedge$ before $\left(t_{1}, t_{2}\right) \supset$ $\exists$ o occurrence-of $(o$, revised-trust $(x, f)) \wedge$ before $E q\left(\operatorname{endof}(o), t_{2}\right) \wedge$ before $\left(t_{1}, \operatorname{endof}(o)\right)$.

T-4: $\forall o, x, f$ occurrence-of $(o$, revised-trust $(x, f)) \supset$ distrusted-in $(x, f$, endof $(o)) \wedge(\exists$ trusted-in $(x, f, t) \wedge$ before $(t$, endof $(o)))$.

Information provided by different sources may also be from different views and as such conflicting. For example, consider the situation where the individual fails a test related to a skill, but gets a positive recommendation from a trusted source on that skill. Assuming that the test is reliable and the recommender is being honest, the discrepancy could be due to factors irrelevant to proficiency in the skill. Of course it could also be the case that the assumptions about trustworthiness and reliability are not accurate or no longer valid. We must take these situations into account when changing the state of a skill statement to refuted using the information provided by a source in the absence of information about actual participation in occurrences of activities enabled by a skill and measurement of related attributes (criteria specified in Section 5). To this end, we specify the reversible relational fluent which takes as input a skill statement and is true if the state of the skill statement has been changed to refuted only based on information provided by a source. In other words, the gold standard for refutation of a skill statement is observation of enabled activities and measurement of related attributes. If the state has been changed to refuted using information other than this, then the state is reversible.

For modifying skill evaluations over time, each source $x$ can either support or reject a particular skill statement $s t$ based on the provided evidence $o$ ( $o$ is used since evidences are occurrences of activities). The state of the skill statement, then, changes using rules R-1 to R-9 depending on whether the source is credible or not. Table 4 summarizes the state transitions based on each rule.

\footnotetext{
${ }^{5}$ Since trust is inherently uncertain, it can be that a source is trusted to a certain degree and distrusted to a certain degree at the same time (Huang and Nicol, 2009). Here, we assume that at a particular point in time a source cannot be both trusted-in and distrusted-in a field of knowledge.

${ }^{6}$ Trust in a source can be adjusted over time as skill statements are demonstrated or refuted, by counting the number of positive and negative experiences among all encounters with that source.
} 
Table 4

Rules used for state transitions.

\begin{tabular}{|l|c|c|c|c|}
\hline From & No Info & Refuted & Possible & Probable \\
\hline No Info & - & R-3 & R-4 & R-1 \\
\hline Refuted & R-6 & - & R-2, R-6.a, R-7 & R-6.b, R-7, R-8 \\
\hline Possible & - & R-3, R-9 & - & R-1, R-8 \\
\hline Probable & R-5 & R-3, R-5.b, R-9 & R-5.a & - \\
\hline
\end{tabular}

If a skill statement is supported by a credible source and it has not yet been demonstrated or refuted, then it is set to state probable:

R-1: $\forall o, x, s t, p, s, l$ supports $(x$, st,$o) \wedge s t=\operatorname{skill-statement}(p, s, l) \wedge x \neq p \wedge$ credible-source-for $(x, s$, endof $(o)) \wedge \neg$ prior (demonstrated $($ st $), o) \wedge$ $\neg \operatorname{prior}(\operatorname{refuted}($ st $), o) \supset$ achieved $(\operatorname{probable}(s t), o)$.

If, on the other hand, st has been refuted but is still reversible, then the state is changed to possible:

R-2: $\forall o, x, s t, p, s, l$ supports $(x, s t, o) \wedge$ st $=\operatorname{skill-statement}(p, s, l) \wedge x \neq p \wedge$ credible-source-for $(x, s$, endof $(o)) \wedge \operatorname{prior}($ refuted $($ st $), o) \wedge \operatorname{prior}($ reversible $($ st $), o) \supset$ (achieved (possible $($ st $), o) \wedge$ falsified(reversible $($ st $), o)$ ).

If a credible source rejects the skill statement then it is set to state refuted. However, this state is reversible if further information is later provided.

R-3: $\forall o, x, s t, p, s, l$ rejects $(x, s t, o) \wedge$ st $=$ skill-statement $(p, s, l) \wedge x \neq p \wedge$ credible-source-for $(x, s$, endof $(o)) \wedge \neg$ prior (demonstrated $(s t), o) \supset$ achieved $($ refuted $($ st $), o) \wedge$ achieved (rever sible $($ st $), o$ ).

In the case of sources which have not yet been verified as credible but are not considered as distrusted, the state is set to possible. This rule is also used in the case of individuals providing information about themselves.

R-4: $\forall o, x, s t, p, s, l, f$ supports $(x, s t, o) \wedge s t=\operatorname{skill-statement}(p, s, l) \wedge$ in-field $(s, f) \wedge$

$(x=p \vee(\neg \operatorname{trusted-in}(x, f$, endof $(o)) \wedge \neg$ distrusted-in $(x, f$, endof $(o)))) \wedge$

$\neg \operatorname{prior}(\operatorname{asserted}($ st $), o) \wedge \neg$ prior $($ refuted $($ st $), o) \supset \operatorname{achieved}($ possible $($ st $), o)$.

As stated earlier, trust in a source may be revised based on the information it provides over time. If a source becomes distrusted, we need to revise the state of the skill statements affected by this change. A skill statement is affected if: 1) information from the source which is now distrusted was used to change its state to either probable or refuted, or 2) trust has been revised in all sources which supported or rejected the skill statement prior to trust in this source being revised. If the state of an affected skill statement has not been changed to demonstrated before trust in the source that asserted it is revised, and another credible source does not exist to support the skill statement, its state is falsified.

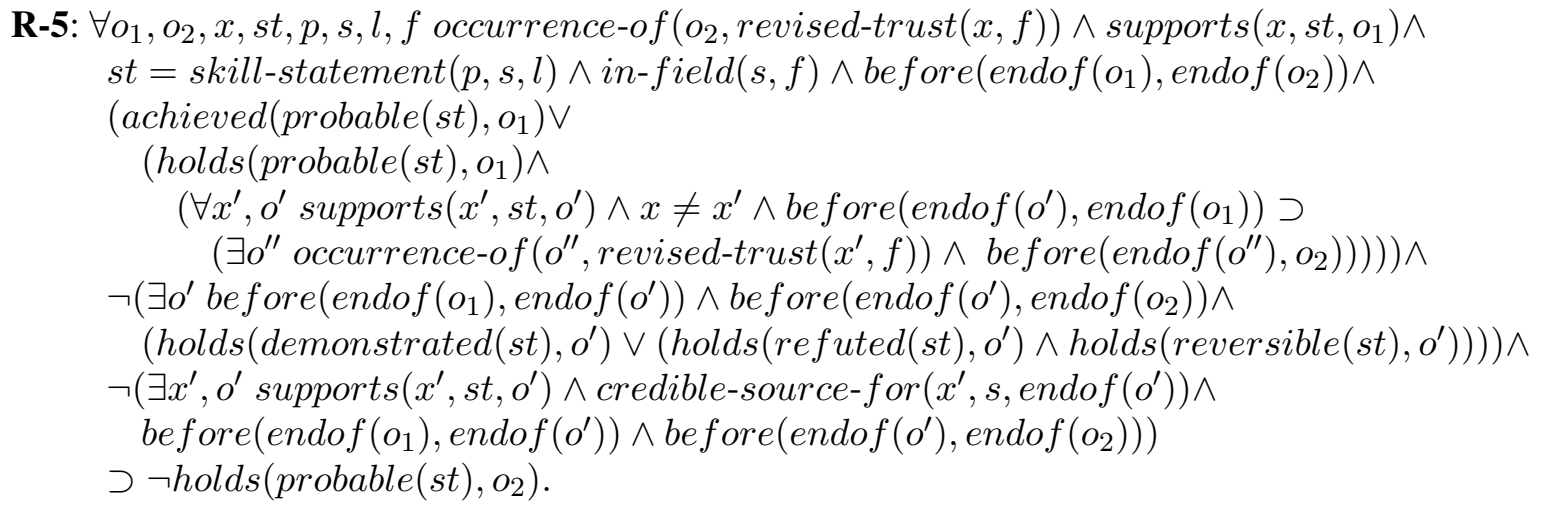


Furthermore, the skill statement needs to be reverted to its prior state if it was in either of the states possible (R-5.a) or refuted but reversible (R-5.b).

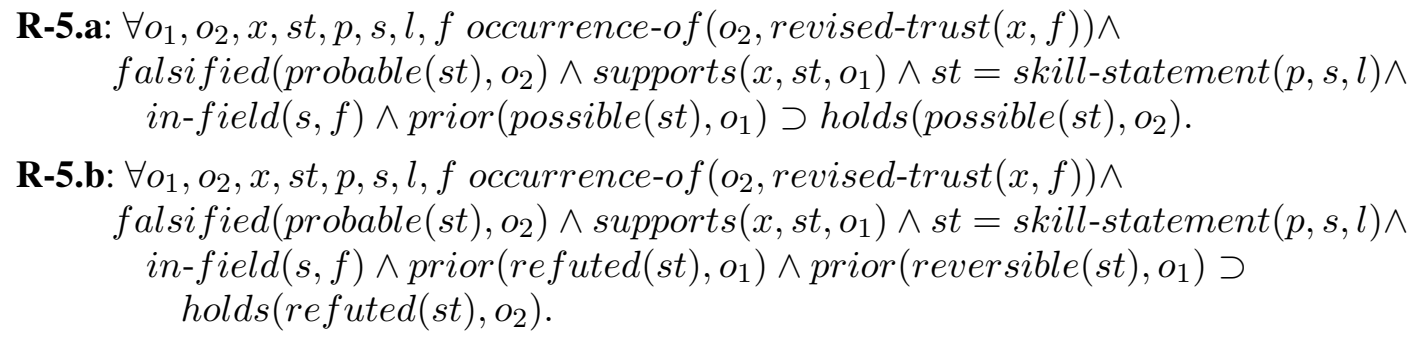

Similarly, if the skill statement was refuted but is still reversible when trust is revised, and no other source exists which rejects this skill statement, then its state is falsified.

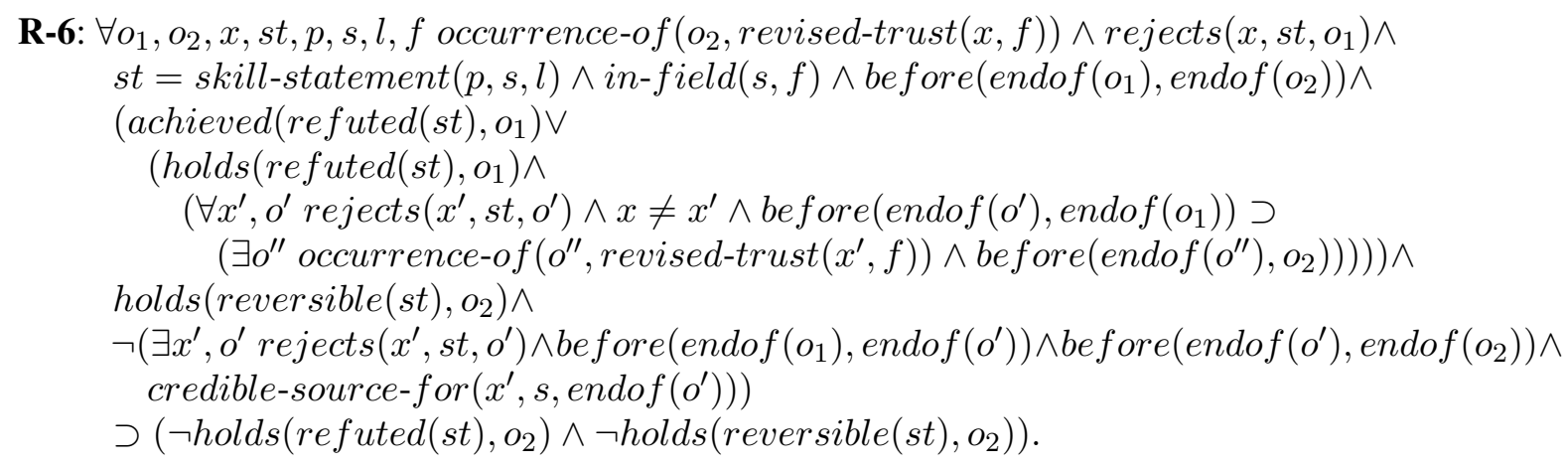

Furthermore, the skill statement needs to be reverted to its prior state if it was in either of the states possible or probable.

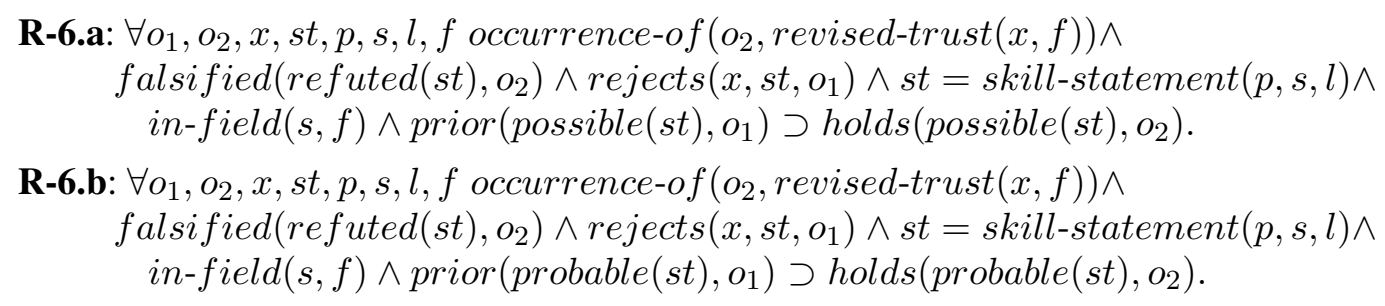

\section{Sources of Skill and Competency Information}

Skill statements can be declared by individuals about themselves or about others, and/or can be derived from 1) activities performed by the individual either online or offline including enrollment in learning activities, experiences related to the workplace, generating content both within the organization and on the Web, and question-answering in online forums; 2) recommendations; 3 ) "wisdom of the crowd"; and 4) assessments in the form of various tests, or evaluations in the form of 360 reviews or performance appraisals (Figure 4). In the remainder of this section we discuss how information from these sources can be used to change the state of skill statements.

A system for skill and competency management should be able to interact and work with other existing IT systems in an organization in order to be more effective. To integrate heterogeneous data from divergent sources about individuals, the incoming data are mapped to an occurrence of a particular activity and added to the knowledgebase. The mapping between the data model of each system and the activities considered is done manually upon the deployment of the system. For example, if the Dublin Core vocabulary (Nilsson et al., 2008) is used by an automated IR agent to annotate content by specifying the creator and subject fields, then we add an occurrence of the activity $\operatorname{tags}(x, c, s)$ denoting agent $x$ (either an individual or an IR agent) tags content $c$ with skill $s$ to the knowledgebase:

$$
\forall p, c, s \text { creator }(c, p) \wedge \operatorname{subject}(c, s) \supset
$$




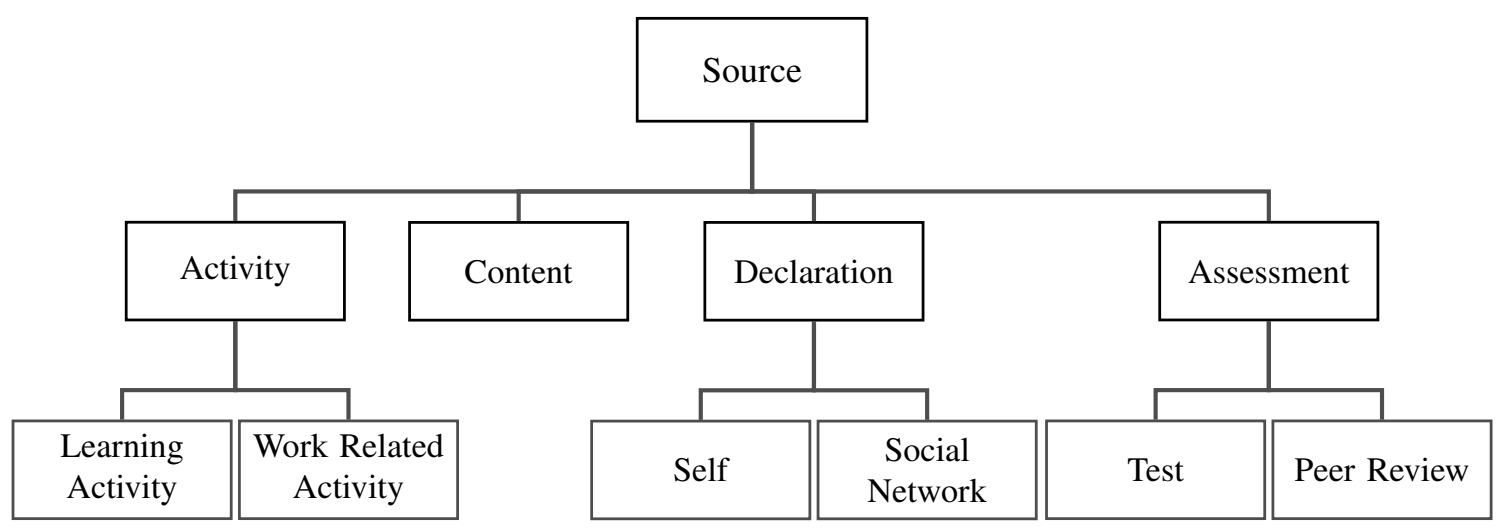

Fig. 4. Sources of skill and competency information

$\exists$ o occurrence-of $(o, \operatorname{tags}(I R$-Agent $, c, s)) \wedge \operatorname{performs}(p, o)$.

Other examples of such activities include declares, learning-activity, and adds-experience. These activities are introduced in the subsequent subsections where appropriate.

\subsection{Declarations of Expertise}

Individuals can declare skill statements about themselves or others. We represent this by two activities declares $(x, p, s, l)$ and declares-neg $(x, p, s, l)$, where agent $x$ is asserting something about the skill $s$ of agent $p$. When $x=p$, the statement is a self-declaration.

I-1: $\forall o, x, p, s, l$ occurrence-of $(o$, declares $(x, p, s, l)) \supset \operatorname{supports}(x, \operatorname{skill-statement}(p, s, l), o)$.

I-2: $\forall o, x, p, s, l$ occurrence-of $(o$, declares-neg $(x, p, s, l)) \supset \operatorname{rejects}(x, \operatorname{skill-statement}(p, s, l)), o)$

Self-declarations of skills and competencies, in particular, have been widely used in many Expertise Finding Systems (Yimam-Seid and Kobsa, 2003). These statements, however, can be incorrect, inaccurate, or insufficient. Others can also declare skills statements about an individual. Skill declarations by others are particularly useful when the recommender is trusted to make judgments about the skill. Considering negative declarations, when the level of proficiency indicated is the lowest possible, it is equivalent to stating that the individual does not have the skill at all.

Figure 5 summarizes the state transition of skill statements based on positive and negative declarations. In particular, self declarations are not considered as credible and as such are assigned to state possible. These declarations are not informative enough to change the state of an already existing skill statement. In the case of positive declaration of skills by others, when a trusted individual gives the recommendation, the state is set to probable according to R-1, otherwise, it is set to possible according to R-4. If a negative declaration is made by someone trusted in the field, then the state of the skill statement is set to refuted according to R-3. However, this evaluation may be reversed.

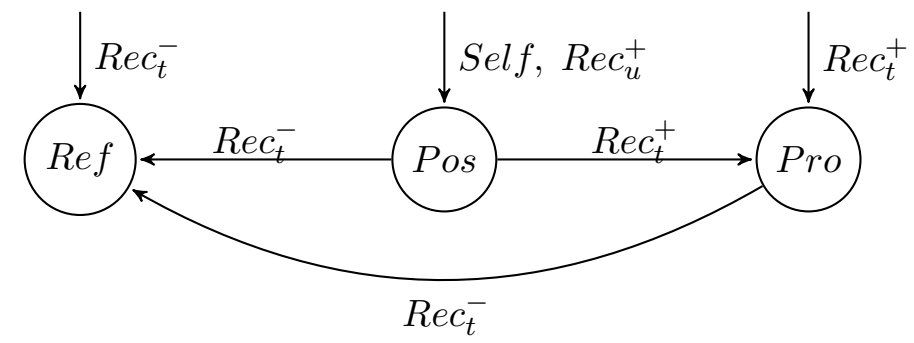

Fig. 5. State diagram for skill declarations. Self represents self declarations, $R e c^{+,-}$represents positive or negative declarations by trusted $(t)$ or untrusted $(u)$ others. 
When relying on other individuals to provide information about an employee, we must note that people have personal goals, change over time, and the nature of their relationships may affect their judgments. In other words, not all sources are honest in exchanging information about others. This means that we cannot rely only on trust relations since the assumption that whoever is trustworthy in previous interactions will be trustworthy in providing information regarding others may not always hold (Qureshi et al., 2010). As such, we need to identify when the information provided by an individual about another can be trusted and when individuals are engaging in collusive behavior. We address this issue by considering the network of relationships that individuals are embedded within. The details of this work are outside the scope of this paper.

\subsection{Learning Activity}

A learning activity (LA) is an activity that has one or more learning outcome associated with it and occurs within a particular context (Conole and Fill, 2005). A learning outcome is what the learners should be able to do after completing the LA. Currently we only consider formal learning activities which occur as a result of instructor-led programs within the curricula of educational institutions or the courses or workshops offered by different agencies (Schugurensky, 2000). This type of evidence also helps in understanding how a skill can be achieved, which is especially useful for arranging training programs.

A formal learning activity (learning-activity) can have a set of skills at a particular proficiency level as required preconditions (has-precondition), but must have at least one skill $s$ at a level of proficiency $l$ as outcome (has-outcome $(a, s, l)$ ). In addition, occurrences of formal learning activities must have occurred at an educational institution:

I-3: $\forall$ a learning-activity $(a) \equiv$

$$
\operatorname{activity}(a) \wedge(\exists s, l \text { skill }(s) \wedge \operatorname{proficiency-level}(l) \wedge \text { has-outcome }(a, s, l)) \text {. }
$$

I-4: $\forall$ o, a learning-activity $(a) \wedge$ occurrence-of $(o, a) \supset \exists$ organization $(x) \wedge$ occurred-at $(o, x)$.

If a skill is both precondition and outcome of a formal learning activity, then the proficiency level of the outcome must be higher.

I-5: $\forall a, s, l_{1}, l_{2}$ has-outcome $\left(a, s, l_{1}\right) \wedge$ has-precondition $\left(a, s, l_{2}\right) \supset$ dominates $\left(l_{1}, l_{2}\right)$.

Having specified formal learning activities, we can define a degree (degree) in a field of study (studyfield) as a program that requires a set of formal learning activities (requires-fla). If an individual has a degree $d$ from an educational institution $x$ (has-degree $(p, d, x)$ ) then it must be the case that he or she has successfully completed all the formal learning activities required for the degree at that institution.

I-6: $\forall p, d, x$ has-degree $(p, d, x) \supset$

$(\forall a$ requires-fla $(d, a) \supset \exists$ occurrence-of $(o, a) \wedge \operatorname{performs}(p, o) \wedge$ occurred-at $(o, x))$.

If an individual has taken a formal learning activity and the skill is a precondition or outcome of it then he or she might have acquired that skill.

I-7: $\forall o, a, p, x$ occurrence-of $(o, a) \wedge$ learning-activity $(a) \wedge$ performs $(p, o) \wedge$ occurred-at $(o, x) \supset$ $(\forall s, l$ (has-outcome $(a, s, l) \vee$ has-precondition $(a, s, l)) \supset$ supports $(x$, skill-statement $(p, s, l), o))$.

Skill statements suggested from taking learning activities, however, are only credible when taken at a reputable institution. Here, we use the notion of system trust, which is the trust placed on the stable or predictable functions or behaviors of a system and includes professional membership-based trust and institutional based trust (Huang and Fox, 2006). The state diagram for formal learning activity is shown in Figure 6. If the formal learning activity was taken at a reputable institution (i.e., the organization trusts the institution), and the skill was not previously demonstrated or refuted, then using R-1 the state is set to probable. Otherwise, if the skill has been refuted, but can be revised, the state is set to possible using R-2. If the learning activity took place at an untrusted institution and the skill has not been asserted or refuted, then the state is set to possible using R-4. 


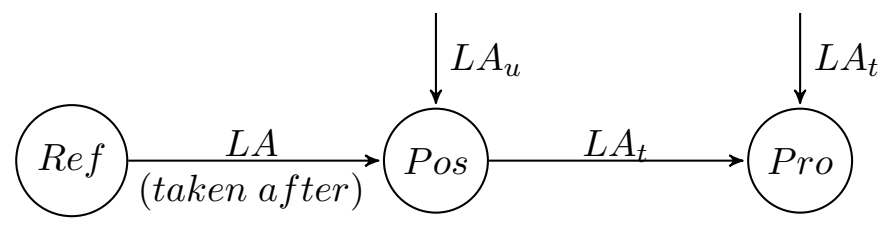

Fig. 6. State diagram for formal learning activity taken at a trusted institution. $L A$ represents formal learning activity taken at a trusted institution $(t)$ or untrusted institution $(u)$.

With learning activities, we need to also consider the case where the individual takes a course after the skill was refuted. If a skill statement has been refuted and the individual takes a learning activity which has the skill as an outcome from a trusted institution, then the individual might have obtained the skill.

R-7: $\forall o, a, p, x$ occurrence-of $(o, a) \wedge$ learning-activity $(a) \wedge$ performs $(p, o) \wedge$ occurred-at $(o, x) \supset$ $\left(\forall s, l_{1}, l_{2}\right.$ has-outcome $\left(a, s, l_{1}\right) \wedge \operatorname{prior}\left(\right.$ refuted $\left(\right.$ skill-statement $\left.\left.\left(p, s, l_{2}\right)\right), o\right) \wedge$

$\left(l_{1}=l_{2} \vee\right.$ dominates $\left.\left(l_{1}, l_{2}\right)\right) \supset\left(\operatorname{achieved}\left(\right.\right.$ possible $\left(\operatorname{skill}\right.$-statement $\left.\left.\left(p, s, l_{1}\right)\right), o\right) \wedge$ supports $\left(x\right.$, skill-statement $\left.\left.\left.\left.\left(p, s, l_{1}\right)\right), o\right)\right)\right)$.

\subsection{Work Experience}

Work experience indicates past performed activities at a particular organization. These are usually indicated by the roles that the individual has played, which define one or more prototypical job functions in that organization. We represent this using the activity adds-experience $(p, r, c)$, where individual $p$ is asserting that they have played role $r$ at organization $c$. These roles and the required skills for those roles can be used to suggest skill statements about the individual. In the Organization Ontology, each role is associated with a set of skills required for the realization of the job functions using the requires-skill relation. We extend this relation to include the minimum level of proficiency required to perform activities related to that role.

To validate individual's previous performance, letters of recommendation are widely used. We represent this using the recommends relation. In this case, the skill statements suggested from work experience are treated as skill declarations by the recommender:

I-8: $\forall o, p, r, c, x, r^{\prime}$ occurrence-of $(o$, adds-experience $(p, r, c)) \wedge$ recommends $(x, p) \wedge$ $\operatorname{plays}\left(x, r^{\prime}, c\right) \supset(\forall s, l$ requires-skill $(r, s, l) \supset \operatorname{supports}(x, \operatorname{skill-statement}(p, s, l), o))$.

If, however, the work experience is not validated by another individual at that organization, then the skill statements are self declarations:

$$
\begin{aligned}
& \text { I-9: } \forall o, p, r, \text { c occurrence-of }(o, \text { adds-experience }(p, r, c)) \wedge \\
& \neg\left(\exists x, r^{\prime} \text { recommends }(x, p) \wedge \text { plays }\left(x, r^{\prime}, c\right)\right) \supset \\
& \quad(\forall s, l \text { requires-skill }(r, s, l) \supset \operatorname{supports}(p, \operatorname{skill-statement}(p, s, l), o)) .
\end{aligned}
$$

Figure 7 shows the state diagram for work experience. Skill statements derived from previous work experience are only credible when verified by a trusted source. In this regard, trust relations can be derived from the hierarchy of roles defined in the Organization Ontology in which role generalization (specialization) are defined through the superior-of (subordinate-of) relations. If the previous work experience is verified by a trusted source and the skill was not demonstrated or refuted previously, then the state is set

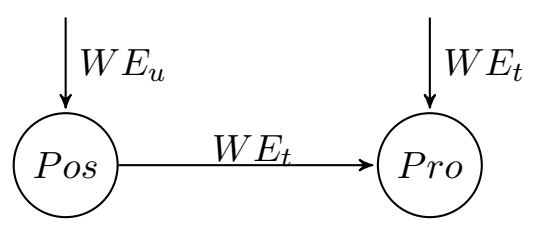

Fig. 7. State diagram for work experience. $W E$ represents work experience with verification from a trusted individual or superior $(t)$ and unverified $(u)$. 
to probable, otherwise, if it was refuted but can be revised, then the state is set to possible. If the previous work experience is not verified, then the state is set to possible similar to self declaration.

\subsection{User Generated Content}

Using content generated by an individual as indicator of expertise has received much attention in previous research. For example, within academic communities, peer-reviewed scientific publications are high quality reliable evidences of expertise. Software codes and documents created in the workplace are other such evidences. With the growth of the Web, there is an additional abundance of publicly available (annotated) user generated content that can be considered. For example, contributing a reliable content to a Wikipedia page indicates that the contributor is knowledgeable on the topic of the page (Stankovic et al., 2010). Corporate blogs are other examples of such user generated content (Kolari et al., 2008). Generated content may also be useful for determining the proficiency level of an individual in the derived skill. For example, considering papers within the academic community, the impact factor of the journal or conference where the paper was published, and the number of citations it has received are factors that indicate a combination of novelty and significance which then indicate proficiency.

An important issue in deriving skill statements based on user generated content is relating documents to domains of expertise and identifying the specific skills that the creator of the content has and the activities that he or she can perform. To relate content to domain of expertise, lexical pattern matching and keyword extraction, and indexing have been considered in previous research (Hansen et al., 2010). The annotation of documents and resources with meta-data using existing vocabularies such as the Dublin Core (Nilsson et al., 2008) by the creator of the document or others through assigning tags to resources (e.g., see the tagging ontologies of (Kim et al., 2008) and (Passant and Laublet, 2008)) for classifying content is another method used. For inferring skill statements, however, relating content to domain of expertise is not enough, and it is also necessary to know if the individual is actually competent enough to perform the required activities. Figure 8 shows examples of types of content that can be used as indicators of expertise. When considering content, two types of documents can be distinguished: 1) documents which suggest the ability to perform activities related to a skill, and 2) documents which only suggest knowledge about a topic and additional information is needed to infer whether an individual can perform specific activities.

Using the Dublin Core vocabulary, we specify content as an object which has a type and an individual (either as a creator or a contributor) associated with it.

$$
\begin{aligned}
\text { I-10: } \forall c \text { content }(c) \equiv & \operatorname{object}(c) \wedge \neg \text { state }(c) \wedge \\
& (\exists x, p \text { content-type }(x) \wedge \operatorname{type}(c, x) \wedge(\text { creator }(c, p) \vee \text { contributor }(c, p))) .
\end{aligned}
$$

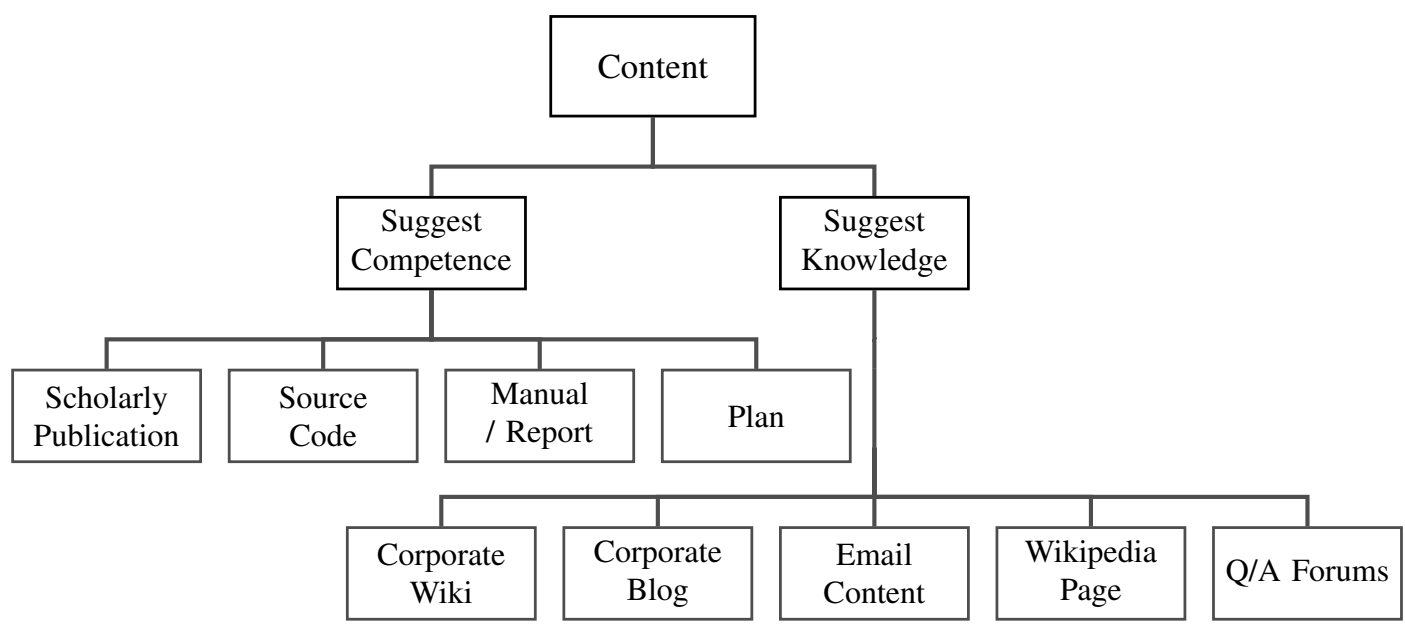

Fig. 8. Example of different types of content that can be used as indicator of expertise 
Documents that suggest competence are the outcome of activities enabled by a skill. More specifically, these outcomes are the reasons for defining the activity in the first place. For example, considering software development, source code is the end result (outcome) of the implementation phase where software engineers program the code for a project. To represent this, we relate activities to the type of content that is produced as a result of participation in an occurrence of that activity. The end result of an activity occurrence, is then, the actual document of that content type.

I-11: $\forall a, x$ activity-outcome $(x, a) \supset$ content-type $(x) \wedge$ activity $(a)$.

I-12: $\forall o, c$ end-result $(c, o) \supset$ content $(c) \wedge$ activity-occurrence $(o)$.

I-13: $\forall$ o, $a, x$ occurrence-of $(o, a) \wedge$ activity-outcome $(x, a) \supset$

$(\exists c$ content $(c) \wedge \operatorname{type}(c, x) \wedge$ end-result $(c, o))$.

If an individual has created a document which was the outcome of an activity, then he or she has participated in an occurrence of that activity:

I-14: $\forall p, c, t, x$ creator $(c, p) \wedge$ type $(c, x) \wedge$ activity-output $(x, a) \supset$ ( $\exists$ o occurrence-of $(o, a) \wedge$ end-result $(c, o) \wedge \operatorname{performs}(p, o))$.

The axioms for demonstration of a skill (S-4 and S-5) apply once the individual has participated in occurrences of all activities enabled by that skill.

When content is not directly related to activities enabled by a skill, it can still be linked to that skill by an information retrieval (IR) technique or tags assigned by other individuals. Tagging data is usually represented as a triple where an agent tags a resource with a particular keyword. We represent this using the activity $\operatorname{tags}(x, c, s)$ denoting agent $x$ (either an individual or an IR agent) tagging content $c$ with skill $s$. When a document is tagged, at the very least some knowledge relevant to the skill can be suggested:

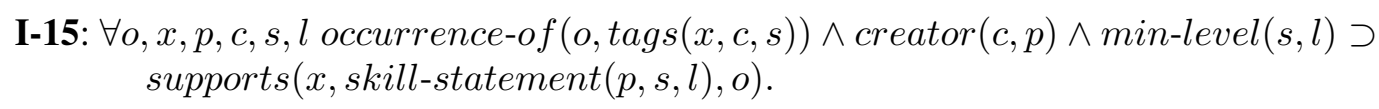

If the agent who tags the document is considered as a credible source, the state is set to probable according to R-1. Otherwise, it is assigned to state possible according to R-4. Note that here the minimum level of proficiency in the related skill is assigned. More accurate proficiency levels can be determined by considering the importance of an individual in the network of all documents created related to that particular knowledge field or skill (i.e., individuals whose work receives many citations can be considered as having higher levels of proficiency). In addition, different mechanisms are usually in place to evaluate user-generated content. In question answering forums, for example, the receiver of the information can provide feedback on the usefulness of the information provided and there is usually a rating of the answer that can be used to imply proficiency. In these forums, the level of proficiency of the information seeker can also be used to determine the proficiency of the information provider. More specifically, if a statement about the same skill exists for the information seeker and she provides positive feedback, then it can be suggested that the information provider has at least the proficiency specified in the information seeker's skill statement. We do not discuss this further in this paper and leave determining proficiency levels from user-generated content more accurately to future work.

\subsection{Assessments}

Assessments can also be used to determine the state of skill statements. In this regard we can consider two types of assessments: 1) peer-review and 2) test.

In many organizations, reviews are performed for evaluating employees' skills. These include performance appraisals, where the employees are reviewed by their immediate managers, and 360-degree reviews, where a combination of self, supervisor, and peer ratings are considered. Tag data can also be viewed as some kind of peer evaluation when the resource tagged is an individual instead of a document (e.g., IBM's Fringe Contacts allows individuals to describe their colleagues by tagging them with keywords on their expertise and interests). When considering peer evaluations, use of multiple raters aver- 
ages out the possible biases of any one member of the group of raters. Feedback and reviews can only be considered as useful and meaningful when reliable, and the use of unreliable ratings on performance and abilities can cause serious problems in the organization (Hensel et al., 2010). Different factors such as how long the rater has known the person, confidentiality of the reviews, and the relation between the rater and the person affect the accuracy of reviews. In a study of accuracy of 360-degree reviews, (Eichinger, 2004) reports that the "known for one to three years" group is the most accurate, and in general reviews by the supervisor are the most accurate followed by peers and direct reports, with self being the least accurate. The supervisor may also have the broadest perspective on the work requirements and be able to take into account shifts in those requirements. (Hensel et al., 2010) reports that although many researchers state that a minimum of three to five peer raters should be used in combination with one supervisor rating, they found that at least ten peers are required for a satisfactory level of reliability.

For this, we consider a voting system in which votes are weighted according to the factors which affect the accuracy of a review such as how long the rater has known the person, confidentiality of the reviews, and the relation between the rater and the person. These weights can be updated over time as individuals interact with one another. We consider two activities votes-for and votes-against and two functions for keeping the count of the number of each for a skill statement: pos-count and neg-count. If an individual votes-for (votes-against) a skill statement then the count is incremented (decremented) by the weight assigned to evaluations by the rater for this individual. Since reviews are usually done periodically, we need to consider how to aggregate the results of different rounds. To this end, the latest reviews are given a higher weight because of freshness, and previous reviews are discounted.

As stated earlier, one of the main complications is that although the more frequent two individuals interact, the more they learn about the competencies of one another, the opportunities to be more biased toward each other also increases (e.g., the type of their personal relationship may affect the accuracy of their reviews). As such, when considering multiple reviews over time by the same person, we need to take this factor into account. If there is a discrepancy between the ratings of a rater in two consequent periods, we compare the recent review with the pool of all reviews at that period: if it is consistent with the majority of the reviews, the weight of the rater increases due to an increase in familiarity with the individual, otherwise, the weight is decreased. These calculations are performed outside of the ontology. Depending on the difference between the two counts, an occurrence of the following activities for the ratee $p$ and skill $s$ is added to the knowledgebase periodically: vote-positively $(p, s, l)$, vote-negatively $(p, s, l)$, and vote-unknown $(p, s, l)$. Based on these occurrences, then, the state of the skill statement changes. If a skill statement is in state possible or refuted but reversible and enough peers vote positively, then the state is changed to probable (R-8). When changing the state to refuted using negative peer reviews, reversible is also set to hold (R-9). Figure 9 shows the state diagram for peer reviews.

R-8: $\forall o, p, s, l$ occurrence-of $(o$, vote-positively $(p, s, l)) \wedge$ $(\operatorname{prior}(\operatorname{possible}(\operatorname{skill-statement}(p, s, l)), o) \vee(\operatorname{prior}(\operatorname{refuted}(\operatorname{skill}$-statement $(p, s, l)), o) \wedge$

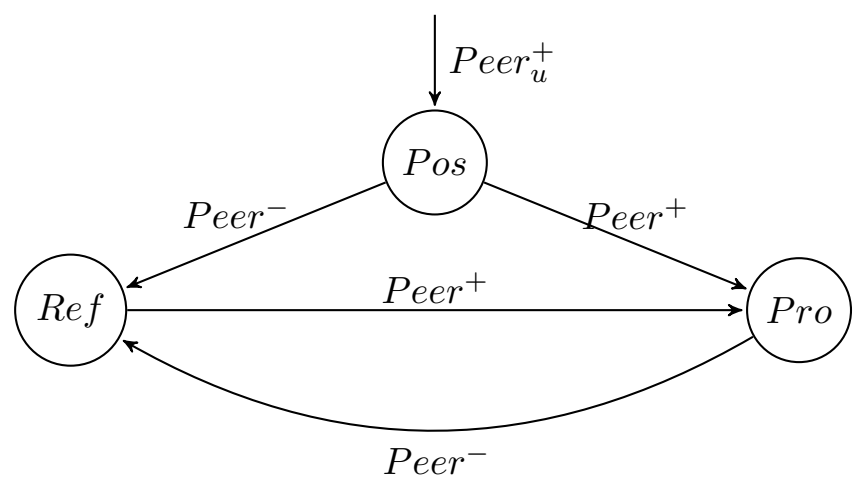

Fig. 9. State diagram for peer reviews. $\mathrm{Peer}^{+}$and $\mathrm{Peer}^{-}$indicate enough positive and negative peer reviews. Peer ${ }_{u}^{+}$indicates generally positive reviews which have not yet reached the threshold. 


$$
\begin{aligned}
& \text { prior }(\text { reversible }(\text { skill-statement }(p, s, l)), o))) \supset \\
& \quad \text { achieved }(\text { probable }(\text { skill-statement }(p, s, l)), o) .
\end{aligned}
$$

R-9: $\forall o, p, s, l$ occurrence-of $(o$, vote-negatively $(p, s, l)) \wedge$

$(\operatorname{prior}(\operatorname{probable}($ skill-statement $(p, s, l)), o) \vee \operatorname{prior}(\operatorname{possible}(\operatorname{skill-statement}(p, s, l)), o)) \supset$ (achieved(refuted $($ skill-statement $(p, s, l)), o) \wedge$ achieved(reversible (skill-statement $(p, s, l)), o))$.

As stated previously, in addition to peer reviews, nowadays many organizations are using different types of online and offline tests for determining and validating individuals' skills. Tests are particularly useful before an individual is hired for filtering the applicant pool. Similar to peer-reviews, tests are meaningful when reliable, i.e. they measure skills correctly. In addition, tests should be valid and unbiased. We define a test as an object which measures the level of proficiency in at least one skill.

I-16: $\forall t$ test $(t) \equiv(\operatorname{object}(t) \wedge \neg$ state $(t) \wedge(\exists s, l$ measures-skill $(t, s, l)))$.

Note that here we are not considering tests which include performing activities related to a particular skill (e.g., job simulations) since those are considered as direct observations for which axioms presented in Section 5 apply.

We consider an activity and two predicates related to tests: takes-test, passes and fails. If an individual passes a test, then he or she probably has the skills which the test measures at a level of proficiency if they were not refuted prior to the test (I-17). If the skill was refuted but is still reversible, then the state is set to possible.

I-17: $\forall o, p, t$ occurrence-of $(o$, takes-test $(p, t)) \wedge \operatorname{passes}(p, t$, endof $(o)) \supset$

$(\forall s, l$ measures-skill $(t, s, l) \supset$ supports $(t, \operatorname{skill-statement}(p, s, l), o))$.

If the individual fails a test, the related skill statements are refuted but can be revised.

I-18: $\forall o, p, t$ occurrence-of $(o$, takes-test $(p, t)) \wedge$ fails $(p, t$, endof $(o)) \supset$ $(\forall s, l$ measures-skill $(t, s, l) \supset$ rejects $(t, \operatorname{skill-statement}(p, s, l), o))$.

\subsection{Other Information}

The profiles can also be improved by considering the taxonomy of skills and highly related skills in the domain of interest. For example, if an individual has demonstrated a skill then it can be derived that he or she has other skills related-to this skill at least at the minimum level of proficiency.

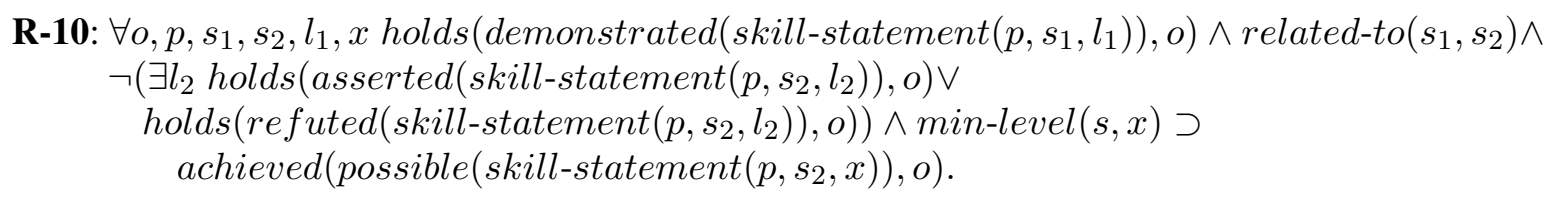

In addition, if an individual has participated in an occurrence of an activity which required a particular tool or technology, then it might be the case that she can perform other activities which require the same resource and as such have the skills that enable those activities. For example, if an individual has experience computing statistics in Microsoft Excel, then she might also be able to do macro programming.

\section{Example}

So far we have discussed how skill and competency information from different sources change the state of skill statements. We now discuss how a combination of these sources can be used to change our belief in skill statements of an individual by giving an example ${ }^{7}$. The overall process is illustrated in Figure 10. The predicates used in this example are repeated in Table 5 for clarity.

\footnotetext{
${ }^{7}$ The updates were verified using the Prover9 theorem prover (McCune, 2005).
} 
Table 5

Predicates used in the example.

\begin{tabular}{|l|l|}
\hline \multicolumn{1}{|c|}{ Predicate } & \multicolumn{1}{c|}{ Informal Definition } \\
\hline enables $(S, A, L)$ & Skill $S$ enables activity $A$ at level of proficiency $L$. \\
\hline requires-value $(S, L, M, X)$ & $\begin{array}{l}\text { Measured attribute } M \text { of skill } S \text { requires-value } X \text { for level of } \\
\text { proficiency } L .\end{array}$ \\
\hline in-field $(S, F)$ & Skill $S$ is in knowledge-field $F$. \\
\hline dominates $\left(L_{1}, L_{2}\right)$ & Proficiency level $L_{1}$ dominates level $L_{2}$. \\
\hline trusted-in $(X, F, T)$ & $\begin{array}{l}\text { Source } X \text { is trusted on producing information in field } F \text { at time } \\
\text { T. }\end{array}$ \\
\hline distrusted-in $(X, F, T)$ & $\begin{array}{l}\text { Source } X \text { is distrusted on producing information in field } F \text { at } \\
\text { time } T \text {. }\end{array}$ \\
\hline has-degree $(P, D, C)$ & Individual $P$ has a degree $D$ from educational institution $C$. \\
\hline requires- $f l a(D, A)$ & Degree $D$ requires formal learning activity $A$. \\
\hline has-outcome $(A, S, L)$ & Formal learning activity $A$ has skill $S$ at level $L$ as outcome. \\
\hline requires-skill $(R, S, L)$ & $\begin{array}{l}\text { Role } R \text { requires skill } S \text { at level } L \text { for the realization of the job } \\
\text { functions. }\end{array}$ \\
\hline plays $(P, R, C)$ & Agent $P$ plays role $R$ at organization $C$. \\
\hline superior $\left(R_{1}, R_{2}\right)$ & This relation establishes a ranking of roles. \\
\hline recommends $(R, P)$ & Individual $R$ recommends $P$. \\
\hline occurrence-of $(O, A)$ & $\begin{array}{l}\text { PSL. Activity-occurrence } O \text { is a particular occurrence of ac- } \\
\text { tivity } A \text {. }\end{array}$ \\
\hline holds $(F, O)$ & PSL. State $F$ is true after the activity-occurrence $O$. \\
\hline declares $(X, P, S, L)$ & Agent $X$ declares that agent $P$ has skill $S$ at level $L$. \\
\hline adds-experience $(P, R, C)$ & Agent $P$ has played role $R$ at organization $C$. \\
\hline
\end{tabular}

Assume a company decides to hire a new employee to fill role $R$ which requires skills $S_{1}, S_{2}$, and $S_{3}$ at levels of proficiency $L_{1,2}, L_{2,3}$, and $L_{3,3}$, respectively, where $L_{i, j}$ refers to the $j$-th level of proficiency associated with skill $i$. The activities that an individual having the skills at these levels are expected to perform and the proficiency scale for each skill is as follows:

- enables $\left(S_{1}, A_{1,1}, L_{1,2}\right) \wedge \operatorname{enables}\left(S_{1}, A_{1,2}, L_{1,2}\right) \wedge \operatorname{enables}\left(S_{1}, A_{1,3}, L_{1,2}\right)$

- requires-value $\left(S_{1}, L_{1,2}, M_{1,1}, X_{1,1}\right)$

- in-field $\left(S_{1}, F_{1}\right)$

- dominates $\left(L_{1,3}, L_{1,2}\right) \wedge$ dominates $\left(L_{1,2}, L_{1,1}\right)$

- enables $\left(S_{2}, A_{2,1}, L_{2,3}\right) \wedge \operatorname{enables}\left(S_{2}, A_{2,2}, L_{2,3}\right)$

- requires-value $\left(S_{2}, L_{2,3}, M_{2,1}, X_{2,1}\right) \wedge$ requires-value $\left(S_{2}, L_{2,3}, M_{2,2}, X_{2,2}\right)$

- in-field $\left(S_{2}, F_{2}\right)$

- dominates $\left(L_{2,3}, L_{2,2}\right) \wedge$ dominates $\left(L_{2,2}, L_{2,1}\right)$

- enables $\left(S_{3}, A_{3,1}, L_{3,3}\right) \wedge \operatorname{enables}\left(S_{3}, A_{3,2}, L_{3,3}\right)$

- requires-value $\left(S_{3}, L_{3,3}, M_{3,1}, X_{3,1}\right)$

- in-field $\left(S_{3}, F_{3}\right)$

- dominates $\left(L_{3,4}, L_{3,3}\right) \wedge$ dominates $\left(L_{3,3}, L_{3,2}\right) \wedge$ dominates $\left(L_{3,2}, L_{3,1}\right)$

The company has asked all potential candidates to create an online profile by declaring their skill set, educational background, and previous work experiences. $P$ is interested in this position and provides the following information for her profile: 


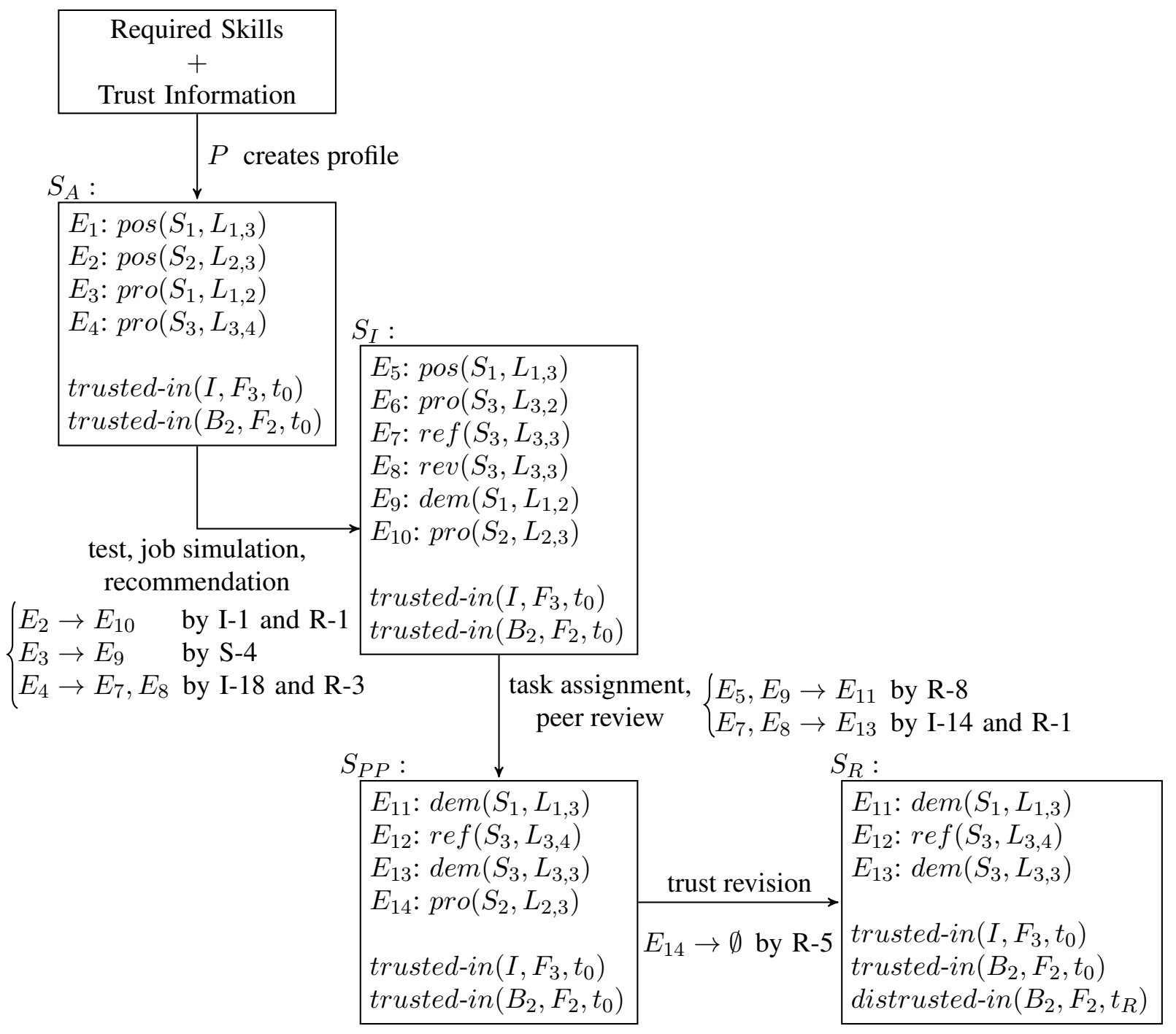

Fig. 10. Example: Modifying skill evaluations over time. $S_{A}, S_{I}, S_{P P}, S_{R}$ represent the state at the end of the application, interview, probation period, and trust revision, respectively. For brevity, only skill statements of individual $P$ that hold and trust in sources of information are included.

- occurrence-of $\left(o_{1}\right.$, declares $\left.\left(P, P, S_{1}, L_{1,3}\right)\right)$

- occurrence-of $\left(o_{2}\right.$, declares $\left.\left(P, P, S_{2}, L_{2,3}\right)\right)$

- has-degree $(P, D, I)$

- occurrence-of $\left(o_{3}, a d d s\right.$-experience $\left.\left(P, R_{1}, C\right)\right)$

- recommends $\left(B_{1}, P\right) \wedge \operatorname{plays}\left(B_{1}, R_{2}, C\right) \wedge \operatorname{superior}\left(R_{2}, R_{1}\right)$

In addition to above, the company has the following information:

- trusted-in $\left(I, F_{3}, t_{0}\right) \wedge$ requires-fla $\left(D, A_{L}\right) \wedge$ has-outcome $\left(A_{L}, S_{3}, L_{3,4}\right)$

- requires-skill $\left(R_{1}, S_{1}, L_{1,2}\right)$

Based on what was presented in the previous sections, the following information is initially known about candidate $P$ :

E-1: holds(possible(skill-statement $\left.\left.\left(P, S_{1}, L_{1,3}\right)\right), o_{1}\right)$.

E-2: holds (possible (skill-statement $\left.\left.\left(P, S_{2}, L_{2,3}\right)\right), o_{2}\right)$.

E-3: holds (probable (skill-statement $\left.\left.\left(P, S_{1}, L_{1,2}\right)\right), o_{3}\right)$.

E-4: holds (probable (skill-statement $\left.\left.\left(P, S_{3}, L_{3,4}\right)\right), o_{4}\right)$. 
E-1 and E-2 are added to the knowledgebase based on self-declarations, E-3 is added based on previous work experience verified by a superior, and E- 4 is added based on learning activity completed at a reputable institution. Note that E-1 and E-3 both hold since $L_{1,3}$ dominates $L_{1,2}$. In other words, it is possible that $P$ has the skill at a higher level, but previous work experience does not support that. We refer to this set as $S_{A}$, representing what the organization believes about the skills of individual $P$ at the time of receiving the application. Note that this set also includes statements at lower levels of proficiency for each skill. For brevity we omit these statements.

Now, assume $P$ is asked to attend an interview consisting of both a written component and a job simulation. The written component tests $S_{1}$ and $S_{3}$ related knowledge at $L_{1,2}$ and $L_{3,3}$ levels, respectively. $P$ passes the part on $S_{1}$ but fails the one for $S_{3}$. This does not change the state of the skill statement on $S_{1}$ since it is already in state probable. However, it does change the state of $S_{3}$ at level $L_{3,3}$ to refuted but reversible. Note that since the skill statement regarding $S_{3}$ was in state probable, a statement was also added for lower levels of proficiency.

For the second part of the interview, $P$ is given a case and asked to implement a solution to it. In this part, it is observed that $P$ can perform activities enabled by $S_{1}$ at $L_{1,2}$ and satisfies the required value for $M_{1}$. Thus, the state of this skill statement (E-3) is changed to demonstrated. $S_{2}$, however, cannot be observed and measured in a short period of time. To get a better understanding regarding this skill, the company asks $B_{2}$, an employee at $C$ whom it trusts in the related field $F_{2}$ to make a recommendation. $B_{2}$ confirms that $P$ has $S_{2}$ at level at least $L_{2,3}$. As such, the state of this skill statement (E-2) is changed to probable. The knowledgebase $\left(S_{I}\right)$ at the end of the interview contains the following statements:

E-5: holds(possible(skill-statement $\left.\left.\left(P, S_{1}, L_{1,3}\right)\right), o_{1}\right)$.

E-6: holds (probable (skill-statement $\left.\left.\left(P, S_{3}, L_{3,2}\right)\right), o_{4}\right)$.

E-7: holds (refuted(skill-statement $\left.\left(P, S_{3}, L_{3,3}\right)\right)$, o $\left.o_{5}\right)$.

E-8: holds (reversible(skill-statement $\left.\left.\left(P, S_{3}, L_{3,3}\right)\right), o_{5}\right)$.

E-9: holds(demonstrated(skill-statement $\left.\left.\left(P, S_{1}, L_{1,2}\right)\right), o_{6}\right)$.

E-10: holds(probable (skill-statement $\left.\left.\left(P, S_{2}, L_{2,3}\right)\right), o_{7}\right)$.

Although $P$ does not satisfy one of the skill requirements $\left(S_{3}\right)$, the company decides that she is the most qualified among the set of candidates. $P$ is hired and given a three months probation period. During this time, her supervisor can closely evaluate her skills by assigning appropriate tasks and monitoring her performance. If at the end of this period it is determined that she is not doing well, then her contract is terminated. Now, assume $P$ 's first project includes activities $A_{1,1}, A_{1,2}, A_{2,1}$, and $A_{3,1}$ which she performs satisfactorily. At the end of the probation period, $P$ gets enough positive reviews for $S_{1}$ at level $L_{1,3}$ from her peers. She also generates content $C_{1}$ which is believed to be the outcome of activity $A_{3,2}$. Hence, it is noted that $P$ has performed all activities enabled by $S_{3}$ at level $L_{3,3}$. At the end of the probation period, her supervisor also notices that $P$ satisfies the required value for $M_{3}$. As such, the state of the skill statement about this skill (E-7) is changed to demonstrated (E-13). E-12 is added since the skill was also refuted at higher levels. It is also noted that the test on $S_{3}$ may not be a credible source for this skill. As such, the knowledgebase $\left(S_{P P}\right)$ representing the state at the end of the probation period contains the following statements:

E-11: holds(demonstrated(skill-statement $\left.\left.\left(P, S_{1}, L_{1,3}\right)\right), o_{1}\right)$.

E-12: holds (refuted(skill-statement $\left.\left.\left(P, S_{3}, L_{3,4}\right)\right), o_{5}\right)$.

E-13: holds(demonstrated(skill-statement $\left.\left(P, S_{3}, L_{3,3}\right)\right)$, $\left.o_{8}\right)$.

E-14: holds (probable(skill-statement $\left.\left.\left(P, S_{2}, L_{2,3}\right)\right), o_{7}\right)$.

The company decides to keep $P$. At time $t_{R}$, however, it finds out that information provided by $B_{2}$ in field $F_{2}$ is not always accurate and so revises trust in $B_{2}$ due to the number of negative encounters having passed a threshold. As such, the following are added to the knowledgebase (by applying T-3):

- distrusted-in $\left(B_{2}, F_{2}, t_{R}\right)$.

- occurrence-of $\left(o_{9}\right.$, revised-trust $\left.\left(B_{2}, F_{2}\right)\right) \wedge \operatorname{endof}\left(o_{9}\right)=t_{R}$. 
This, in turn, triggers state revisions for skill statements affected by this change. Since $B_{2}$ is the only source for (E-14), this skill statement is falsified (and, therefore, the company has no information about $S_{2}$ of $P$ at this point in time). Later on, it is noticed that $P$ cannot in fact perform all activities enabled by $S_{2}$ satisfactorily. As such, the skill is refuted at this level and $P$ is asked to enroll in a training program.

\section{Evaluation}

The ontology was evaluated from four perspectives: 1) Does the ontology's axioms entail the competency questions, 2) is the ontology consistent, 3) does it adequately represent the skills and competencies of human resources in a real-world domain, and 4) is reasoning with the ontology scalable.

\subsection{Entailment of Competency Questions}

The ontology presented in Sections 4-7 represents and answers all of the competency questions of Section 3.1. In particular, using the terms described in Section 4, informal competency questions 1-4 can be formally expressed. These questions are simple consequences of the axioms and our representation of skills and proficiency. Competency questions 5-8 are formally represented using the terminology developed in Sections 5-7. In the following we discuss each competency question one by one. See Appendix 2 for a detailed discussion of each competency question.

\subsection{Consistency}

Ensuring that an ontology is consistent is an important step in the development and evaluation of ontologies since an inconsistent ontology can be used to deduce arbitrary conclusions (Baclawski et al., 2002). Consistency is proved by showing that the ontology has a model. In such a model, however, some relations may have empty extensions. Non-trivial consistency is proved by ensuring that every relation in the ontology has a nonempty extension by adding existential sentences requiring the existence of domain entities for making every relation true (Hahmann, 2013). For checking the consistency of the ontology, we constructed non-trivial models using the Mace4 (McCune, 2005) model builder. Given the axioms and the additional existential sentences, Mace 4 constructed models for all but the Sources of Information module ${ }^{8}$.

\subsection{Prototype Decision Support System}

The ontology was implemented at Novator Systems in a prototype HR decision support system whose function was to match individuals to a set of job requirements and allow competency gap analysis. The prototype includes a web interface that allows individuals to upload their resumes, declare their skill set, educational background, and work experiences, and provide reviews about their peers. The skill domain at Novator Systems consists of 96 technical computer skills related to all aspects of software design and development, programming, database design and administration, documentation, software testing, web design and development, etc. It also includes 112 skills related to specific tools or technologies. Six employees participated in the evaluation of the system, declaring 3 to 31 skills each. The DSS used the Prover9 theorem prover (McCune, 2005) to construct individuals' expertise profiles and match them to job requirements. The results were reviewed by the head of HR and the six participants. The main complication in the design and development of the system was to have employees realize that the system is to facilitate expert finding and help improve and not to criticize or undermine their skills and abilities. This was done by adding information boxes which explained the purpose of each feature. The participants highlighted the following features as being important:

\footnotetext{
${ }^{8}$ Prover9, however, detected no inconsistencies within the specified time limit of one hour.
} 
- relating skills to activities enables the organization to link skills and competencies to organizational goals,

- identification of individual's skills based on their educational background and work experiences eliminates the need to explicitly declare each separately,

- specifying attributes such as recency allows for better matching individuals to positions based on their current qualifications,

- providing peer reviews at the skill level allows a more fine-grained assessment and evaluation of individual's expertise.

\subsection{Scalability}

An important issue regarding the actual use of any information system is scalability. A close look at the nature of instances populating our ontology reveals that it is not necessary to reason over all existing instances since the data can be partitioned into independent sets. More specifically, consider the three different types of data required for inferring and validating expertise profiles: 1) data about activities and skills within a particular domain, 2) expertise profiles and data about the activities each individual performs, and 3) data about trust and credibility of sources. Considering the first type of data, we can consider an upper bound on the number of activities and skills within a particular domain. If an organization operates over multiple domains, we can consider them as separate partitions. Considering the second type of data, we note that expertise profiles of individuals are independent of one another and can be stored separately for each human resource. There is also an upper bound on the number of work-related skills each individual can have. LinkedIn, for example, allows individuals to declare a maximum of 50 skills. Data on trust and credibility of sources can be arbitrarily large. However, not all of this data is necessary for reasoning about skills and competencies of an individual. For each source that states a skill statement, we can ask a query and store the related trust information alongside each expertise profile. Taking the partitioning into account, the approach presented in this paper will scale in a real world context in terms of the information related to one person.

\section{Conclusions}

The focus of this paper has been on the representation of and reasoning about skills and competencies over time. This work was motivated by the need to have accurate and well defined data about human resources in order to more effectively manage competencies and find experts. To stay competitive within the market, organizations need to accurately grasp the competency of their human resources. This is particularly important for most services organizations, especially those with a large number of employees which provide a variety of products and services to multiple and changing clients. Wrong decisions in this regard may result in significant loss of value and human resources.

In this paper, we developed a formal ontology for representing and reasoning about skills and competencies in a dynamic environment. The first contribution of this work is the presentation of a clearer definition of skill and proficiency and their formalization in first-order logic. We related proficiency levels to the span of activities that one can perform in addition to measurable attributes related to skills in order to specify what can be expected of someone who possess a skill. Our approach to the representation of skills and competencies has a number of advantages. Defining skills in terms of the performance of activities and measurable attributes improves competency measurement and evaluation by ensuring a consistent interpretation of the meaning of proficiency. Moreover, it enables the automatic confirmation or refutation of individual's competencies based on their participation in activities and measurement of the related attributes. Furthermore, it allows us to use the process specification language (PSL), which has a rigorous well-defined formal syntax and semantics, as the representation language.

Taking an ontological approach to the problem of profiling experts also allows us to address problems related to heterogeneous information sources and inter-operability. The second contribution of this work 
is the development of a technique for inferring and validating skills and competencies by integrating data from different sources of information, and stating how skill evaluations are modified over time using information from these sources. More specifically, starting with less than accurate models of individuals, additional skills are inferred based on the activities individuals participate in and the contents they generate. The competencies are then validated over time by using assessments, "wisdom of the crowd", and peer-reviews. In other words, skill evaluation changes over time using the axioms as new information becomes available. This also provides the means for modifying trust in the sources of skill and competency information over time as observations are made and skills are demonstrated or refuted.

The main evaluation criterion for the development of the ontology in this paper was competency, the capability of a representation to support tasks for which it is designed. This was done by specifying a set of questions which the ontology should be able to represent and answer using a necessary and sufficient set of axioms, if it contains the relevant information. An interesting topic of future research is to evaluate the expert profiles by measuring how close they are to reality. We plan to design simulations and user studies to better understand under what circumstances the models converge into the real evaluation and how important a source is in increasing the quality of the information that is known. Cost of gaining information is another important issue that must be considered in future work. In addition, further work should be done on evaluating the usefulness of the models in different domains and studying how structure within a particular domain relates to the general model.

Individual's social network and peers are important sources of skill and competency information. In relying on these sources, however, we must consider that people have personal goals, change over time, and the nature of their relationships may affect their judgments. In other words, not all sources are honest in exchanging information about others even if they have been trustworthy in the past. As such, we need to identify dishonest behavior over time and use this information to update trust in the sources of information. There are other situations as well in which we may need to update trust in some sources. If a trusted source is consistently providing incorrect information then it is necessary to modify trust in that source. Furthermore, in real world settings, most of the information provided, especially at the beginning, may be from untrusted sources. Untrusted sources are mainly useful for stating the possibility of having a skill, and are not very useful for state transitions. We can adjust trust in the sources of skill and competency information over time as observations are made and skill statements are demonstrated or refuted.

In most organizations individuals are usually members of teams set up to pursue specific projects. Teams are temporary in nature and are set up when needed. Although we did not consider teams in this paper, we believe most of the concepts and relations defined for individuals can be extended to be associated with teams. When considering individuals for teams, however, complexities may arise due to fitness between an individual and other team members. Furthermore, project requirements, personal and technical characteristics of human resources and their availability are important factors that influence the success of a team. It would be interesting to see how these complexities can be supported by automated techniques.

\section{Acknowledgements}

The authors gratefully acknowledge the constructive comments of Michael Gruninger. This research is supported in part by the Natural Science and Engineering Research Council of Canada.

\section{References}

Baclawski, K., Kokar, M.M., Waldinger, R., Kogut, P.A. (2002). Consistency Checking of Semantic Web Ontologies, Proceedings of the First International Semantic Web Conference, 454-459.

Balog, K., Azzopardi, L., \& de Rijke, M. (2006). Formal models for expert finding in enterprise corpora, Proceedings of the 29th annual international ACM SIGIR Conference on Research and Development in Information Retrieval, Seattle, Washington, ACM, 43-50.

Becerra-Fernandez, I. (2006). Searching for Experts on the Web: A Review of Contemporary Expertise Locator Systems, $A C M$ Transactions on Internet Technology, 6(4), 333-355. 
Bernstein, M., Tan, D., Smith, G., Czerwinski, M., Horvitz, E. (2009). Collabio: A Game for Annotating People Within Social Networks, Proceedings of UIST 2009, ACM Press, 97-100.

Biesalski, E., \& Abecker, A. (2006). Skill-Profile Matching with Similarity Measures, Proceedings of the 8th International Conference on Enterprise Information Systems (ICEIS), Paphos, Cyprus, 210-18.

Bizer, C., Heese, R., Mochol, M., Oldakowski, R., Toksdorf, R., \& Eckstein, R. (2005). The Impact of Semantic Web Technologies on Job Recruitment Processes, Proceedings of the 7th Internationale Tagung Wirtschaftsinformatik, 1367-1383.

Bizer, C., Heath, T., Idehen, K., \& Berners-Lee, T. (2008). Linked data on the web. Proceedings of the 17th International Conference on World Wide Web, Beijing, China, ACM, 1265-1266.

Braun, S., \& Schmidt, A. (2008). People Tagging \& Ontology Maturing: Towards Collaborative Competence Management, Proceedings of the 8th International Conference on the Design of Cooperative Systems (COOP), Carry-le-Rouet, France, Springer, 133-âĂŞ154.

Campion, M.A., Fink, A.A., Ruggeberg, B.J., Carr, L., Phillips, G.M., \& Odman, R.B. (2011). Doing Competencies Well: Best Practices in Competency Modeling, Personnel Psychology, 64, 225-262.

Cao, Y., Liu, J., Bao, S., \& Li, H. (2005). Research on Expert Search at Enterprise Track of TREC 2005, Proceedings of the 14th Text REtrieval Conference, Gaithersburg, Maryland, National Institute of Standards and Technology (NIST).

Colucci, S., Di Noia, T., Di Sciascio, E., Donini, F. M., Mongiello, M., \& Mottola, M. (2003). A Formal Approach to OntologyBased Semantic Match of Skills Descriptions, Journal of Universal Computer Science 9(12), 1437-1454.

Colucci, S., Di Noia, T., Di Sciascio, E., Donini, F. M., \& Ragone, A. (2007). Semantic-based Skill Management for Automated Task Assignment and Courseware Composition, Journal of Universal Computer Science 13(9), 1184-1212.

Conole, G., Fill, K. (2005). A Learning Design Toolkit to Create Pedagogically Effective Learning Activities, Journal of Interactive Media in Education, 8.

D'Amore, (2008). Expert Finding in Disparate. Environments. Doctoral Thesis, Department of Information Studies, University of Sheffield, Sheffield, United Kingdom.

De Coi, J., Herder, E., Koesling, A., Lofi, C., Olmedilla, D., Papatreou, O., et al. (2007). A Model for Competence Gap Analysis, Proceedings of the 3rd International Conference on Web Information Systems and Technologies (WEBIST), Barcelona, Spain.

Dittmann, L. (2003). Towards Ontology-based Skills Management Project KOWIEN Report: University Duisburg-Essen.

Dorn, J., Naz, T., \& Pichlmair, M. (2007). Ontology Development for Human Resource Management, Proceedings of the 4th International Conference on Knowledge Managements, 109-120.

Draganidis, F., \& Mentzas G. (2006). Competency Based Management: A Review of Systems and Approaches, Information Management \& Computer Security, 14(1), 51-64.

Draganidis, F., Chamopoulou P., \& Mentzas G. (2008). An Ontology Based Tool for Competency Management and Learning Paths, Proceedings of $i$-KNOW'06.

Draganidis, F., Chamopoulou P., \& Mentzas G. (2008). A Semantic Web Architecture for Integrating Competence Management and Learning Paths, Journal of Knowledge Management, 12(6), 121-136.

Earl, M. (2001). Knowledge Management Strategies: Toward a Taxonomy, Journal of Management Information Systems, 18(1), $215-233$.

Eichinger, R. (2004). Patterns of Rater Accuracy in 360-degree Feedback. Perspectives, 27, $23-25$.

Farrell, S., Lau, T. (2006). Fringe Contacts: People-Tagging for the Enterprise, Proceedings of the Collaborative Web Tagging Workshop at WWW'06.

Fazel-Zarandi, M., \& Yu, E. (2008). Ontology-Based Expertise Finding, Proceedings of the 7th International Conference on Practical Aspects of Knowledge Management, Lecture Notes in Computer Science, Yokohama, Japan.

Fazel-Zarandi, M.; Fox, M.S. (2009). Semantic Matchmaking for Job Recruitment: An Ontology-Based Hybrid Approach, Proceedings of the 3rd International Workshop on Service Matchmaking and Resource Retrieval in the Semantic Web at the 8th International Semantic Web Conference (ISWC 2010), Washington D.C., USA.

Fazel-Zarandi, M., \& Fox, M. S. (2012). An Ontology for Skill and Competency Management, Proceedings of the 7th International Conference on Formal Ontologies in Information Systems (FOIS), Graz, Austria.

Fernandez-Araoz, C., Groysberg, B., \& Nohria, N. (2009). The Definitive Guide To Recruiting in Good Times and Bad, Harvard Business Review, 87 (5): 74-84.

Fox, M. S., Barbuceanu, M., \& Gruninger, M. (1996). An Organisation Ontology for Enterprise Modeling: Preliminary Concepts for Linking Structure and Behaviour, Computers in Industry, 29(1-2), 123-134.

Fox, M. S., \& Gruninger, M. (1998). Enterprise Modelling, AI Magazine, 109-121.

Gomez-Perez, A., Ramirez, J., \& Villazon-Terrazas, B. (2007). Reusing Human Resources Management Standards for Employment Services, Proceedings of the Workshop on the First Industrial Results of Semantic Technologies (FIRST) at ISWC/ASWC, 28-41.

Gruninger, M., Fox, M. S. 1995. 'Methodology for the design and evaluation of ontologies.' Workshop on Basic Ontological Issues in Knowledge Sharing at IJCAI-1995.

Gruninger, M., \& Menzel, C. (2003). Process Specification Language: Theory and Applications, AI Magazine, 24, 63-74.

Gu, Y., Soutchanski, M. (2010). A Description Logic Based Situation Calculus, Annals of Mathematics and Artificial Intelligence, 58(1-2), 3-83.

Hahmann, T. (2013). A Reconciliation of Logical Theories of Space: From Multidimensional Mereotopology to Geometry, PhD Thesis, University of Toronto.

Hansen, D. L., Khopkar, T., \& Zhang, J. (2010). Recommender Systems and Expert Locators, Encyclopedia of Library and Information Sciences, Third Edition, 4433-4441.

Hensel, R., Meijers, F., van der Leeden, R., \& Kessels, J. (2010). 360 Degree Feedback: How Many Raters are Needed for Reliable Ratings on the Capacity to Develop Competences, With Personal Qualities as Development Goals?, The International Journal of Human Resource Management, 21(15), 2813-2830. 
Herling, R. W. (2000). Operational Definitions of Expertise and Competence, Advances in Developing Human Resources, 2(8), $8-21$.

Hilligoss, B., \& Rieh, S.O. (2008). Defining a Unifying Framework for Credibility Assessment: Construct, Heuristics and Interaction in Context, Information Processing and Management, 44, 1476-âĂŞ1484

HR-XML, HR-XML Consortium Competencies (2006), Retrieved January 15, 2012 from http://ns.hr-xml.org/2_5/HR-XML2_5/CPO/Competencies.html.

Huang, J. (2008). Knowledge Provenance: An Approach to Modeling and Maintaining the Evolution and Validity of Knowledge. Doctoral Thesis, Department of Mechanical and Industrial Engineering, University of Toronto, Toronto, Canada.

Huang, J., \& Nicol, D. (2009). A Calculus of Trust and Its Application to PKI and Identity Management, Proceedings of the 8th Symposium on Identity and Trust on the Internet, Gaithersburg, MD, USA, 23-27.

Huang, J., \& Fox, M. S. (2006). An Ontology of Trust - Formal Semantics and Transitivity, Proceedings of the 8th International Conference on Electronic Commerce, Fredericton, New Brunswick, Canada, 259-270.

Huang, Y., Contractor, N., \& Yao, Y. (2008). CI-KNOW: recommendation based on social networks, Proceedings of the 2008 International Conference on Digital Government Research, Montreal, Canada, 27-33.

IEEE RCD, IEEE Draft Standard for Learning Technology - Standard for Reusable Competency Definitions (2004), Retrieved January 15, 2012, from http://ltsc.ieee.org/wg20/files/IEEE_1484.20.WD_01_rough.pdf.

IMS RDCEO, IMS Reusable Definition of Competency or Educational Objective (2002), Retrieved January 15, 2012, from http://www.imsglobal.org/competencies/index.html.

Jarrar, M., Vervenne, L., \& Maynard, D. (2007). HR-Semantics Roadmap - The Semantic Challenges and Opportunities in the Human Resources Domain, Technical Report.

Katsumi, M., \& Gruninger, M. (2010) Theorem Proving in the Ontology Lifecycle, Proceedings of Proceedings of the International Conference on Knowledge Engineering and Ontology Development (KEOD), 37-49.

Kim, H., Passant, A., Breslin, J. G., Scerri, S., \& Decker, S. (2008). Review and Alignment of Tag Ontologies for Semanticallylinked Data in Collaborative Tagging Spaces, Proceedings of the 2nd International Conference on Semantic Computing, San Francisco, USA.

Kim, H. M., Fox, M. S., \& Gruninger, M. (1999). An Ontology for Quality Management - Enabling Quality Problem Identification and Tracing, BT Technology Journal, 17(4), 131-140.

Kodama, M. (2007). Project-based Organization in the Knowledge-based Society, Imperial College Press.

Kolari, P., Finin, T., Lyons, K., \& Yesha, Y. (2008). Expert Search using Internal Corporate Blogs, Proceedings of the Workshop on Future Challenges in Expertise Retrieval at SIGIR 2008, 2-5.

Kuhn, W. (2010). Modeling vs Encoding for the Semantic Web, Semantic Web, 1, 1-5.

Ley, T., Albert, D., \& Lindstaedt, S. (2007). Competency Management using the Competence Performance Approach: Modeling, Assessment, Validation, and Use, Competencies in Organizational E-Learning: Concepts and Tools, Idea Group Inc., 83119.

Liyanage, S. (2007). Competency Management and Systems, Competencies in Organizational E-Learning: Concepts and Tools, Idea Group Inc, 10-40.

McCune, W. (2005 - 2010). Prover9 and Mace4, http://www.cs.unm.edu/m̃ccune/Prover9.

Marsh, S. P., \& Debben, M. R. (2005). Trust, Untrust, Distrust and Mistrust - An Exploration of the Dark(er) Side, Proceedings of iTrust2005, Lecture Notes in Computer Science, 3477, 17-33.

Martin, D., Burstein, M., McDermott, D. McIlraith, S., Paolucci M., Sycara, K., McGuinness, D.L., Sirin, E. \& Srinivasan, N. (2007). Bringing Semantics to Web Services with OWL-S, Proceedings of World Wide Web, 243-277.

Maybury, M. T. (2006). Expert Finding Systems, MITRE Center for Integrated Intelligence Systems, Bedford, Massachusetts, USA.

McClelland, D. C. (1973). Testing for Competency rather than Intelligence, American Psychologist, 28, 1-14.

McPhie, N.A.G. 2010. Job Simulations: Trying Out for a Federal Job, DIANE Publishing Company.

Mochol, M., Wache, H., \& Nixon, L. (2007). Improving the Accuracy of Job Search with Semantic Techniques, Proceedings of the 10th International Conference on Business Information Systems, Poznan, Poland: Springer, 301-313.

Moyer, R. (2001). Defining and Measuring Competencies: An Overview of Approaches (2001) Click2learn Inc.

Naveh, Y., Richter, Y., Altshuler, Y., Gresh, D.L., Connors, D.P. (2007). Workforce Optimization: Identification and Assignment of Professional Workers using Constraint Programming, IBM Journal of Research and Development - Business optimization, 51(3), 263-279.

Nicklin, J.M., Roch, S.G. (2009). Letters of Recommendation: Controversy and Consensus from Expert Perspectives, International Journal of Selection and Assessment, 17(1), 76-91.

Nilsson, M., Powell, A., Johnston, P., \& Naeve, A. (2008). Expressing Dublin Core metadata using the Resource Description Framework (RDF), from http://www.dublincore.org/documents/dc-rdf/

Passant, A., \& Laublet, P. (2008). Meaning of a Tag: A Collaborative Approach to Bridge the Gap Between Tagging and Linked Data, Proceedings of the WWW 2008 Workshop Linked Data on the Web (LDOW2008), Beijing, China.

Prins, F. J., Nadolski, R. J., Berlanga, A. J., Drachsler, H., Hummel, H. G. K., \& Koper, R. (2008). Competence Description for Personal Recommendation: The Importance of Identifying the Complexity of Learning and Performance Situations, Educational Technology \& Society 11(3), 141-152.

Qureshi, B., Min, G., \& Kouvatsos, D. (2010). Collusion Detection and Prevention with FIRE+ Trust and Reputation Model. Proceedings of the 10th International Conference on Computer and Information Technology (CIT'2010) (Bradford, United Kingdom), 2548-2555.

Sampson, D. G., \& Fytros, D. (2008). Competence Models in Technology-Enhanced Competence-Based Learning, Handbook of Information Technologies for Education and Training, 155-177.

Sampson, D. G. (2009). Competence-Related Metadata for Educational Resources that Support Lifelong Competence Development Programmes, Educational Technology \& Society 12(4), 149-159. 
Sanchez-Alonso, S., \& Frosch-Wilke D. (2007). An Ontological Representation of Competencies as Codified Knowledge, Competencies in Organizational E-Learning, 169-184.

Schmidt, A., \& Kunzmann, C. (2006). Towards a Human Resource Development Ontology for Combining Competence Management and Technology-Enhanced Workplace Learning, Lecture Notes in Computer Science 4278, 1078-1087.

Schmidt, A., \& Kunzmann, C. (2007). Sustainable Competency-Oriented Human Resource Development with Ontology-Based Comptency Catalogs, eChallenges.

Schugurensky, D. (2000). The Forms of Informal Learning: Towards a Conceptualization of the Field, NALL Working Paper No. 19.

Self, C. S. (1996). Credibility, An Integrated Approach to Communication Theory and Research, Mahwah, NJ: Lawrence Erlbaum Associates, 421 Ü-441.

Seta, K., Ikeda, M., Hirata, K., Hayashi, Y., Kuriyama, K. (2005). A Human Resource Model and Evidence Based EvaluationOntology for IT Skill Standards-, Proceeding of the 2005 Conference on Towards Sustainable and Scalable Educational Innovations Informed by the Learning Sciences, 388-395.

Sicilia, M. (2005). Ontology-Based Competency Management: Infrastructures for the Knowledge Intensive Learning Organization, Intelligent Learning Infrastructure for Knowledge Intensive Organizations: A Semantic Web Perspective, IGI Global, 302-324.

Stankovic, M., Wagner, C., Jovanovic, J., \& Laublet, P. (2010). Looking for Experts? What can Linked Data do for You?, Proceedings of the WWW Workshop Linked Data on the Web (LDOW), Raleigh, NC.

Stevens G.W. (2012). A Critical Review of the Science and Practice of Competency Modeling, Human Resource Development Review.

Tommasi, G.W., Williams, K.B., Nordstrom, C.R. (1998). Letters of Recommendation: What Information Captures HR Professionals' Attention?, Journal of Business and Psychology, 13(1), 5-18.

Tseng, S., \& Fogg, B. J. (1999). Credibility and Computing Technology, Communications of the ACM, 42(5), 39-44.

Urquiza, A. (2007). A Survey of Competency Management Software Information Systems in the Framework of Human Resources Management, Competencies in Organizational E-Learning: Concepts and Tools, 41-82.

Yimam-Seid, D., \& Kobsa, A. (2003). Expert-Finding Systems for Organizations: Problem and Domain Analysis and the DEMOIR Approach, Journal of Organizational Computing and Electronic Commerce 13(1), 1-24.

\section{Appendix 1 - Lexicon}

\subsection{Skill Measurement Module}

\begin{tabular}{|l|l|}
\hline \multicolumn{1}{|c|}{ Predicate } & \multicolumn{1}{c|}{ Informal Definition } \\
\hline set $(X)$ & Sets are objects with at least one member. \\
\hline interval $(X)$ & Intervals are objects with min and max values. \\
\hline measured-attribute $(M)$ & A measurable attribute related to a skill. \\
\hline measurement-unit $(U)$ & Unit of measurement for a measured-attribute. \\
\hline specification-set $(S p)$ & $\begin{array}{l}\text { A set of values which denotes possible values for a measured- } \\
\text { attribute. }\end{array}$ \\
\hline proficiency-level $(L)$ & $\begin{array}{l}\text { Refers to the ranking of the ability of an individual to perform } \\
\text { the activities enabled by a particular skill. }\end{array}$ \\
\hline set-member $(X, Y)$ & Object $X$ is a member of set $Y$. \\
\hline in-interval $(X, Y)$ & $X$ is in interval $Y$. \\
\hline has-spec $(M, S p)$ & Measured-attribute $M$ has specification set $S p$. \\
\hline has-unit $(M, U)$ & Measured-attribute $M$ has unit of measurement $U$. . \\
\hline sless-than $(X, Y)$ & $\begin{array}{l}\text { This relation is used to impose an ordering on members of a } \\
\text { set. }\end{array}$ \\
\hline lesser $(X, Y)$ & This relation is used to impose an ordering on an interval. \\
\hline dominates $\left(L_{1}, L_{2}\right)$ & Proficiency level $L_{1}$ dominates level $L_{2}$. \\
\hline
\end{tabular}




\begin{tabular}{|l|l|}
\hline \multicolumn{1}{|c|}{ Predicate } & \multicolumn{1}{c|}{ Informal Definition } \\
\hline skill $(S)$ & $\begin{array}{l}\text { A class or type of skill. A skill suggests the possibility of per- } \\
\text { forming an activity. }\end{array}$ \\
\hline knowledge-Field $(F)$ & A field of knowledge. \\
\hline enables $(S, A, L)$ & Skill $S$ enables activity $A$ at level of proficiency $L$. \\
\hline enabling-suite $(S, A, L)$ & $\begin{array}{l}\text { A complex activity that includes all the activities enabled by a } \\
\text { skill at a particular level of proficiency. }\end{array}$ \\
\hline in-field $(S, F)$ & Skill $S$ is in knowledge-field $F$. \\
\hline related-to $\left(S_{1}, S_{2}\right)$ & $\begin{array}{l}\text { Two skills are related if they enable the same activity, or if } \\
\text { they enable different subactivities of the same activity. }\end{array}$ \\
\hline requires-value $(S, L, M, X)$ & $\begin{array}{l}\text { Skill } S \text { at level of proficiency } L \text { for measured attribute } M \text { re- } \\
\text { quires value } X .\end{array}$ \\
\hline subskill-of $\left(S_{1}, S_{2}\right)$ & $\begin{array}{l}\text { This relation indicates skill specialization, forming a taxon- } \\
\text { omy of skills. }\end{array}$ \\
\hline
\end{tabular}

\subsection{Organization and Trust Ontologies Modification}

\begin{tabular}{|l|l|}
\hline \multicolumn{1}{|c|}{ Predicate } & \multicolumn{1}{c|}{ Informal Definition } \\
\hline requires-skill $(R, S, L)$ & Role $R$ requires skill $S$ at level of proficiency $L$. \\
\hline remmends $(X, Y)$ & Organization-agent $X$ recommends organization-agent $Y$. \\
\hline credible-source-for $(X, S, T)$ & $\begin{array}{l}\text { Source } X \text { is a credible source of information for skill } S \text { at } \\
\text { timepoint } T \text {. }\end{array}$ \\
\hline
\end{tabular}

\subsection{Skill Statement Module}

\begin{tabular}{|l|l|}
\hline \multicolumn{1}{|c|}{ Predicate } & \multicolumn{1}{c|}{ Informal Definition } \\
\hline demonstrated(skill-statement $(p, s, l))$ & $\begin{array}{l}\text { Relational fluent. } p \text { has demonstrated skill } s \text { at level of pro- } \\
\text { ficiency at least } l \text {. }\end{array}$ \\
\hline probable(skill-statement $(p, s, l))$ & $\begin{array}{l}\text { Relational fluent. It is highly probable that } p \text { has skill } s \text { at } \\
\text { level of proficiency at least } l \text {. }\end{array}$ \\
\hline possible $($ skill-statement $(p, s, l))$ & $\begin{array}{l}\text { Relational fluent. It is possible that } p \text { has skill } s \text { at level of } \\
\text { proficiency at least } l \text {. }\end{array}$ \\
\hline refuted $($ skill-statement $(p, s, l))$ & Relational fluent. skill-statement $(p, s, l)$ has been refuted. \\
\hline asserted $($ skill-statement $(p, s, l))$ & $\begin{array}{l}\text { Relational fluent. } \text { skill-statement }(p, s, l) \text { is demonstrated, } \\
\text { probable, or } \text { possible. }\end{array}$ \\
\hline reversible(skill-statement $(p, s, l))$ & $\begin{array}{l}\text { Relational fluent. skill-statement }(p, s, l) \text { is reversible if its } \\
\text { state has changed to refuted using information other than } \\
\text { direct observation. }\end{array}$ \\
\hline
\end{tabular}

\subsection{Sources of Information Module}




\begin{tabular}{|l|l|}
\hline \multicolumn{1}{|c|}{ Predicate } & \multicolumn{1}{c|}{ Informal Definition } \\
\hline supports $(X, S t, O)$ & $X$ supports skill statement $S t$ with evidence $O$. \\
\hline rejects $(X, S t, O)$ & $X$ rejects skill statement $S t$ with evidence $O$. \\
\hline performs $(P, O)$ & $P$ performs activity-occurrence $O$. \\
\hline declares $(X, P, S, L)$ & Activity. Agent $X$ declares that agent $P$ has skill $S$ at level L. \\
\hline declares-neg $(X, P, S, L)$ & $\begin{array}{l}\text { Activity. Agent } X \text { declares that agent } P \text { does not have skill } S \\
\text { at level L. }\end{array}$ \\
\hline learning-activity $(A)$ & $\begin{array}{l}\text { Activity. An activity which has at least one skill at a level of } \\
\text { proficiency as outcome. }\end{array}$ \\
\hline has-outcome $(A, S, L)$ & The outcome of a learning activity. \\
\hline has-precondition $(A, S, L)$ & $\begin{array}{l}\text { Preconditions of a learning activity, these are skills which an } \\
\text { individual should have in order to take the activity. }\end{array}$ \\
\hline degree $(D)$ & An object that requires a set of formal learning activities. \\
\hline requires- $f l a(D, A)$ & $D$ requires formal learning activity $A$. \\
\hline has-degree $(P, D, C)$ & $P$ has degree $D$ from educational institution $C$. \\
\hline adds-experience $(P, R, C)$ & Activity. Agent $P$ has played role $R$ at organization $C$. \\
\hline content $(X)$ & $\begin{array}{l}\text { An object with an individual either as a creator or a contributor } \\
\text { associated with it. }\end{array}$ \\
\hline content-type $(X)$ & A class or type of content. \\
\hline activity-outcome $(X, A)$ & Content-type $X$ is the outcome of activity $A$. \\
\hline end-result $(X, O)$ & Content $X$ is the end result of activity-occurrence $O$. \\
\hline tags $(X, Y, S)$ & Activity. $X$ tags content $Y$ with skill $S$. \\
\hline test $(X)$ & $\begin{array}{l}\text { An object which measures the level of proficiency in at least } \\
\text { one skill. }\end{array}$ \\
\hline measures-skill $(X, S, L)$ & Test $X$ measures skill $S$ at level of proficiency $L$. \\
\hline takes-test $(P, X)$ & Activity. Agent $P$ takes test $X$. \\
\hline passes $(P, X, T)$ & $P$ passed test $X$ at timepoint $T$. \\
\hline fails $(P, X, T)$ & $P$ failed test $X$ at timepoint $T$. \\
\hline
\end{tabular}

\section{Appendix 2 - Entailment of the Competency Questions}

In this section, we repeat the competency questions presented in Section 3.2 and discuss how the ontology represents and answers these questions.

Q-1 What skills are needed to perform the required activities?

Given activity $A$, this question can be formally represented as:

$\exists s$ enables $(s, A, l)$.

Q-2 Are two skills related?

Given skills $S_{1}$ and $S_{2}$, this question can be formally represented as:

related-to $\left(S_{1}, S_{2}\right) \vee$ subskill-of $\left(S_{1}, S_{2}\right)$.

Q-3 What are the proficiency reference levels for evaluation against a skill?

Given skill $S$, this question can be formally represented as:

$\exists l \operatorname{enables}(S, a, l)$. 
Q-4 What are the criteria for determining whether an individual possesses a skill at a level of proficiency? Given skill $S$, level of proficiency $L$, measured-attribute $M$, and measurement unit $U$, the following questions can be formally represented.

Q-4.1 What are the activities that the individual should be able to perform?

$\exists$ a enables $(S, a, L)$.

Q-4.2 What are the attributes related to that skill that can be measured?

$\exists m$ requires-value $(S, L, m, x)$.

Q-4.3 What is the unit of measurement for an attribute related to a skill?

$\exists$ u requires-value $(S, L, m, x) \wedge$ has-unit $(m, u)$.

Q-4.4 What ought to be the measured value to be ranked at a level of proficiency?

$\exists x$ requires-value $(S, L, M, x)$.

Q-5 What evidence suggests that an individual has a skill at a level of proficiency?

Given individual $P$, skill $S$, and level of proficiency $P$, this question can be formally represented as:

$\exists$ o achieved (asserted (skill-statement $(P, S, L)), o) \wedge(\operatorname{participates-in}(P, o) \vee \operatorname{per} f o r m s(P, o))$.

If a skill statement is asserted, or, in other words, in any of the states demonstrated, probable, or possible, then a source supporting it is suggesting that the individual has the skill. We consider evidences as occurrences of activities. The evidence can be suggested by the performance of activities enabled by a skill using axioms S-4 and S-5, or by different sources of skill and competency information using one of the axioms R-1, R-2, R-4, R-6-R-8, or R-10.

Q-6 Which source is providing this evidence? Is it a credible source of information?

Given individual $P$, skill $S$, and level of proficiency $P$, this question can be formally represented as:

$\exists x$ supports $(x$, skill-statement $(P, S, L), o) \wedge($ participates-in $(P, o) \vee \operatorname{performs}(P, o)) \wedge$ credible-source-for $(x, S$, endof $(o))$.

Q-7 What are the skill statements about an individual at a given point in time?

Given individual $P$ and timepoint $T$, the following questions can be formally represented.

Q-7.1 What are the demonstrated skills of an individual at a given time point?

$\exists$ s holds(demonstrated(skill-statement $(P, s, l)), o) \wedge$ beforeEq $(\operatorname{endof}(o), T) \wedge$

$\neg\left(\exists o^{\prime} h o l d s\left(\right.\right.$ refuted $($ skill-statement $\left.(P, s, l)), o^{\prime}\right) \wedge$ beforeE $q\left(\operatorname{endof}(o)\right.$, endof $\left.\left(o^{\prime}\right)\right) \wedge$ before $\left.E q\left(\operatorname{endof}\left(o^{\prime}\right), T\right)\right)$.

The state of a skill statement can change to demonstrated only if the individual performs all the activities enabled by a skill and satisfies all the measured attributes as specified by S- 4 . However, if a person fails a measured-attribute later on due to knowledge decay, the state can change to refuted using S-6. All the other axioms will not change the state of a demonstrated skill statement.

Q-7.2 What are the suggested skills of an individual at a given time point, i.e. those skills that have not been observed but the individual may possess?

$\exists s($ holds $($ probable $($ skill-statement $(P, s, l)), o) \vee \operatorname{holds}(\operatorname{possible}(\operatorname{skill}$-statement $(P, s, l)), o))$ $\wedge$ before $E q($ endof $(o), T) \wedge \neg\left(\exists o^{\prime}\left(\right.\right.$ holds $\left(\right.$ refuted $($ skill-statement $\left.(P, s, l)), o^{\prime}\right) \vee$

holds (demonstrated (skill-statement $\left.\left.(P, s, l)), o^{\prime}\right)\right) \wedge$

before $E q\left(\operatorname{endof}(o)\right.$, endof $\left.\left(o^{\prime}\right)\right) \wedge$ before $\left.E q\left(\operatorname{endof}\left(o^{\prime}\right), T\right)\right)$.

The state of a skill statement can change to probable if the individual has performed all the enabled activities but has not been measured yet using S-5, or using R-1, R-6-R-7. Similarly, the state of a skill statement can change to possible using R-2, R-4-R-7. However, if a person performs all the activities enabled by a skill and satisfies all the measured attributes later on, then the state change 
to demonstrated using S-4. Additionally, axioms R-3, R-5, and R-9 change the state of a probable and/or possible skill statement to refuted. All the other axioms will not change the state of a probable or possible skill statement.

Q-7.3 What are the refuted skills of an individual at a given time point, i.e. those skills that the individual does not have?

$\exists$ s holds(refuted(skill-statement $(P, s, l)), o) \wedge$ before $E q(\operatorname{endof}(o), T) \wedge$

$\neg\left(\exists o^{\prime} h o l d s\left(\right.\right.$ asserted $($ skill-statement $\left.(P, s, l)), o^{\prime}\right) \wedge$ beforeE $q\left(\operatorname{endof}(o), \operatorname{endof}\left(o^{\prime}\right)\right) \wedge$ before $\left.E q\left(\operatorname{endof}\left(o^{\prime}\right), T\right)\right)$.

The state of a skill statement can change to refuted if the individual fails at least on measuredattribute as specified by S- 6 or through negative assertions by a source of skill and competency information using one of the axioms R-3, R-5, or R-9. However, if a person performs all the activities enabled by a skill and satisfies all the measured-attributes later on, then the state change to demonstrated using S-4. Additionally, axioms R-2, R-6-R-8 change the state of a refuted skill statement to probable or possible. All the other axioms will not change the state of a refuted skill statement.

Q-8 How did belief in a skill statement change over time?

Given individual $P$, skill $S$, and level of proficiency $P$, this question can be formally represented as:

$\exists o, t$ (holds(demonstrated(skill-statement $(P, S, L)), o) \vee$

holds (probable (skill-statement $(P, S, L)), o) \vee$

holds (possible(skill-statement $(P, S, L)), o) \vee$

holds $($ refuted $(\operatorname{skill-statement}(P, S, L)), o)) \wedge \operatorname{endof}(o)=t$.

The history of how belief in a skill statement has changed over time can be retrieved by querying the knowledgebase for the occurrences which affected a particular skill statement along with the ending timepoint of each occurrence. 